Pacific

Journal of

Mathematics

\title{
SMALL EXTENSIONS OF WITT RINGS
}

\author{
Robert W. Fitzgerald
}




\title{
SMALL EXTENSIONS OF WITT RINGS
}

\author{
ROBERT W. FitzGERALD
}

\begin{abstract}
We consider certain Witt ring extensions $S$ of a noetherian Witt ring $R$ obtained by adding one new generator. The conditions on the new generator are those known to hold when $R$ is the Witt ring of a field $F, S$ is the Witt ring of a field $K$ and $K / F$ is an odd degree extension. We show that if $R$ is of elementary type then so is $S$.
\end{abstract}

The elementary type conjecture is a proposed classification of noetherian Witt rings. A potential source of counter-examples is as follows: Start with a field $F$ where $W F$ is known (necessarily of elementary type) then look at noetherian $W K$ for extension fields $K$ of $F$. Jacob and Ware [3] have shown that $W K$ is again of elementary type when $[K: F]=2$. Here we look at the simplest case of odd degree extensions, again showing $W K$ is of elementary type. We note that $W F$ is noetherian iff $G(F) \equiv F^{\cdot} / F^{\cdot 2}$ is finite. Also when $K / F$ has odd degree then $G(F) \cong F^{\cdot} K^{\cdot 2} / K^{\cdot 2}$ embeds into $G(K)$.

We will in fact work with abstract Witt rings $R$ (as defined by Marshall [4]) with associated group of one dimensional forms $G(R)$. The small extensions considered here are as follows. Let $H$ be a subgroup of $G(R)$. We say a Witt ring $S$ is an $H$-extension of $R$ if there exists an $\alpha \in G(S)$ such that:

(1) $G(S)=\{1, \alpha\} G(R)$, and

(2) For all $x \in G(R)$ we have:

$$
\begin{gathered}
D_{S}\langle 1,-x\rangle= \begin{cases}D_{R}\langle 1,-x\rangle, \\
\{1, \alpha\} D_{R}\langle 1,-x\rangle & \text { if } x \notin H\end{cases} \\
D_{S}\langle 1,-\alpha x\rangle=\{1,-\alpha x\}\left(D_{R}\langle 1,-x\rangle \cap H\right) .
\end{gathered}
$$

These conditions hold for $R=W F, S=W K$ when $K / F$ is an odd degree extension and $[G(K): G(F)]=2$ by $[\mathbf{2}, 4.7]$ (we note that $[\mathbf{2}, 4.7]$ should include the condition that $N_{K / F}(a)=1$ ). No such field extensions are known. However, there are many examples of $H$-extensions of abstract Witt rings, which we determine inductively. This can be viewed as a first step in classifying extensions of noetherian Witt rings. It also helps the search for odd degree extensions $K / F$ with $[G(K): G(F)]=2$, while lessening the motivation for such a search. 
The proof of the main result is a long series of technical lemmas, only one of which (3.1) has independent interest. However, most of the results mimic the expected behavior of the field extension case, evidence the elementary type conjecture holds. This is speculative since there may be no field extensions yielding $H$-extensions. However, we indulge in this suggestive speculation once, after (1.5).

For any group $H, H$ denotes $H \backslash\{1\}$. The quaternionic mapping associated to $R$ will be denoted by $q$. For $x \in G(R), Q(x)=\{q(x, y): y \in G(R)\}$ and for a subgroup $H, Q(H)=\{q(h, y): h \in H, y \in G(R)\}$. The value set of $\langle 1,-x\rangle$ is $D\langle 1,-x\rangle=\{y \in G(R): q(x, y)=1\}$. We will often work with several Witt rings at once and write $q_{R}, Q_{R}(x)$ and $D_{R}\langle 1,-x\rangle$ to indicate these objects for $R$.

$R$ is of local type if $|q(G(R), G(R))|=2$. We let $E_{n}$ denote the elementary 2 -group of order $n$. The group ring $R\left[E_{n}\right]$ is again a Witt ring. An element $t \in G(R)$ is rigid if $D\langle 1, t\rangle=\{1, t\}$ and $t$ is birigid if both $t$ and $-t$ are rigid. The basic part of $R, B(R)$, consists of \pm 1 and all $x \in G(R)$ with either $x$ or $-x$ not rigid. $B(R)$ is a subgroup of $G(R)$ and $R=R_{0}[G(R) / B(R)]$, where $R_{0}$ is the Witt ring generated by $B(R)$. We express this last statement by writing $R_{0}=W(B(R))$.

The product in the category of Witt rings is:

$$
R_{1} \sqcap R_{2}=\left\{\left(r_{1}, r_{2}\right): r_{i} \in R_{i} \quad \text { and } \quad \operatorname{dim} r_{1} \equiv \operatorname{dim} r_{2} \quad(\bmod 2)\right\} .
$$

If $R=R_{1} \sqcap R_{2}$ then $G(R)=G\left(R_{1}\right) \times G\left(R_{2}\right)$ and:

$$
D_{R}\langle(1,1),(x, y)\rangle=D_{R_{1}}\langle 1, x\rangle \times D_{R_{2}}\langle 1, y\rangle .
$$

The radical of $R$ is $\operatorname{rad}(R)=\{x \in G(R): D\langle 1,-x\rangle=G(R)\}$. We say $R$ is degenerate if $\operatorname{rad}(R) \neq 1$ and totally degenerate if $\operatorname{rad}(R)=G(R) . D_{n}$ denotes a totally degenerate Witt ring with square class group of order $2^{n}$. There are two possibilities for $D_{n}$ depending on whether -1 is a square or not. Specifically, $D_{n}$ is either a product of $n$ copies of $(\mathbb{Z} / 2 \mathbb{Z})\left[E_{1}\right]$ or $n$ copies of $\mathbb{Z} / 4 \mathbb{Z}$. If $R$ is degenerate then there exist uniquely determined $n$ and nondegenerate Witt ring $R_{0}$ such that $R=D_{n} \sqcap R_{0} . R_{0}$ is the non-degenerate part of $R$.

$R$ is of elementary type if it can be built from $\mathbb{Z} / 2 \mathbb{Z}, \mathbb{Z} / 4 \mathbb{Z}$ and Witt rings of local type by a succession of group ring extensions (for some $E_{n}$ ) and products. The elementary type conjecture is that every noetherian Witt ring is of elementary type.

\section{Group ring extensions.}

To help the reader navigate the following six sections of lemmas, we point out here the highlights. We will prove $S$ is of elementary type by induction on $|G(R)|$. If $R$ is of local type then generally $H=1$ or $G(R)$ and $S$ is determined (2.3), (1.1). The exceptions occur when $R=L_{2,0}$ (there are 
three possible $H$ 's) and when $R=L_{2,1}$ (four possible $H$ 's). The resulting extensions are determined in (1.5).

If $R=R_{0}\left[E_{n}\right]$ is a group ring either $H$ is one of three special subgroups or $S=S_{0}\left[E_{n}\right]$ with $S_{0}$ an $H$-extension of $R_{0}$ (1.5). If $R=R_{1} \sqcap R_{2}$ is a product then $S$ is generally a product of one factor with an $H$-extension of the other factor $(5.7),(6.1)$. There are exceptional cases when $R_{1}=\mathbb{Z}$ (5.9) or $R_{1}$ is totally degenerate (4.1).

Lemma 1.1. Let $S$ be an $H$-extension of $R$.

(a) If $H=1$ then $S=R\left[E_{1}\right]$, with $E_{1}$ generated by $\alpha$.

(b) If $H=G(R)$ then $S=D_{1} \sqcap R$, with $D_{1}$ generated by $\alpha$.

Proof. Suppose first that $H=1$. Then for all $g \in G(R)$ we have from the definition of an $H$-extension that $D_{S}\langle 1,-\alpha g\rangle=\{1,-\alpha g\}\left(D_{R}\langle 1,-g\rangle \cap H\right)$ and so $\alpha g$ is birigid. Thus $B(S) \subset G(R)$ and $G(S)=\{1, \alpha\} G(R)$. So by [4, 5.19] $S=R\left[E_{1}\right]$, where $E_{1}$ is generated by $\alpha$.

Next suppose that $H=G(R)$. Then $D_{S}\langle 1,-\alpha\rangle=\{1,-\alpha\}\left(D_{R}\langle 1,-1\rangle \cap\right.$ $H)=G(S)$. Hence $\alpha \in \operatorname{rad}(S)$. Then by [4, pp. 105-106] $S=D_{1} \sqcap R$, where $D_{1}$ is generated by $\alpha$.

When $H \neq 1$, which we will often assume in light of (1.1), we use the following notation (recall that $B(R)$ is the basic part of $R$ ):

$$
\begin{aligned}
T & =\bigcup_{h \in H^{\cdot}} D_{R}\langle 1,-h\rangle \\
T_{0} & =\bigcup_{h \in H^{\cdot}}\left(D_{R}\langle 1,-h\rangle \backslash\{-h\}\right) \\
B(H) & =H \cap B(R) \\
B T & =\bigcup_{h \in B(H)} D_{R}\langle 1,-h\rangle \\
B T_{0} & =\bigcup_{h \in B(H)}\left(D_{R}\langle 1,-h\rangle \backslash\{-h\}\right) .
\end{aligned}
$$

Lemma 1.2. Let $S$ be an $H$-extension of $R$ with $|H|>1$. Then:

(a) $\pm T_{0} \subset B(R) \subset \pm T$,

(b) $B(S)= \pm\{1, \alpha\} T$.

Proof. First note that $D_{S}\langle 1,-\alpha\rangle=\{1,-\alpha\} H$, and $|H|>1$ imply $\alpha \in B(S)$. If $x \in G(R) \backslash \pm T$ then $D_{R}\langle 1, \pm x\rangle \cap H=\{1\}$ so by the definition of $H$ extensions, $D_{S}\langle 1, \pm \alpha x\rangle=\{1, \pm \alpha x\}$. Hence $\alpha x \notin B(S)$, and as $\alpha \in B(S)$, $x \notin B(S)$. That is, $x$ is birigid in $S$, and so also in $R$. Thus $x \notin B(R)$ and we have:

$$
\begin{aligned}
& B(R) \subset \pm T, \\
& B(S) \subset \pm\{1, \alpha\} T .
\end{aligned}
$$


Let $x \in T_{0}, x \neq-1$ so that for some $h \in H^{*}, x \in D_{R}\langle 1,-h\rangle$ and $x \neq-h$. Then $D_{R}\langle 1,-x\rangle$ contains $-x, h$ which are distinct and not equal to 1 . So $-x \in B(R)$ and also $x \in B(R)$. If $x=-1$ then again $x \in B(R)$. Thus $T_{0} \subset B(R)$, and so $\pm T_{0} \subset B(R)$ completing the proof of (a).

If $x \in T$ with $x \in D_{R}\langle 1,-h\rangle, h \in H^{\cdot}$ then

$$
D_{S}\langle 1,-x\rangle=\{1,-\alpha x\}\left(D_{R}\langle 1,-x\rangle \cap H\right)
$$

contains $\{1,-\alpha x, h,-\alpha x h\}$. Thus $-\alpha x \in B(S)$. Again $\alpha \in B(S)$ so $x \in$ $B(S)$. This shows $\pm\{1, \alpha\} T \subset B(S)$, completing the proof of (b).

Lemma 1.3. If $S$ is an $H$-extension of $R$ with $|H|>1$ then $B(R)= \pm B T$ and either:

(a) $B(R)= \pm B(H)$ and $B(S)= \pm\{1, \alpha\} H$, or

(b) $B(R)= \pm T$ and $B(S)=\{1, \alpha\} B(R)$.

Proof. If $h \in H \backslash B(H)$ then $D_{R}\langle 1,-h\rangle=\{1,-h\}$. So:

$$
\pm T= \pm B T \cup \pm(H \backslash B(H)) .
$$

Now $B(R) \subset \pm T$ by (1.2)(a) and $B(R) \cap \pm(H \backslash B(H))=\emptyset$ so $B(R) \subset \pm B T$. Conversely,

$$
\pm B T= \pm(B T \cup B(H)) \subset \pm T_{0} \cup \pm B(H) \subset B(R),
$$

which proves the first statement.

Now (1.4) gives:

$$
\pm T= \pm B T \cup \pm H=B(R) \cup \pm H
$$

and $(1.2)(b)$ gives:

$$
B(S)= \pm\{1, \alpha\} T=\{1, \alpha\} B(R) \cup \pm\{1, \alpha\} H .
$$

This expresses the group $B(S)$ as the union of two subgroups, hence either:

(i) $\{1, \alpha\} B(R) \subset \pm\{1, \alpha\} H$, or

(ii) $\pm\{1, \alpha\} H \subset\{1, \alpha\} B(R)$.

In case (i) $B(S)= \pm\{1, \alpha\} H$ and $B(R) \subset \pm\{1, \alpha\} H \cap G(R)= \pm H$. Hence $B(R)= \pm B(H)$. In case (ii) $B(S)=\{1, \alpha\} B(R)$ and $H \subset B(R)$. Then $H=B(H), B T=T$ and by the first statement $B(R)= \pm T$.

Recall that any Witt ring $R$ can be written as $R_{0}[G(R) / B(R)]$, where $R_{0}=W(B(R))$, the Witt ring generated by $B(R)$. See $[\mathbf{4}$, Chapter 5 , Section 7] for details.

Proposition 1.5. Let $R=R_{0}\left[E_{n}\right]$, with $R_{0}$ basic. Let $S$ be an $H$-extension of $R$. Then:

(a) If $H=1$ then $S=R_{0}\left[E_{n+1}\right]$.

(b) If $|H|>1$ and $H \subset G\left(R_{0}\right)$ then $S=S_{0}\left[E_{n}\right]$, for some Witt ring $S_{0}$ that is an $H$-extension of $R_{0}$ (with the same $\alpha$ ). 
(c) If $H \not \subset G\left(R_{0}\right)$ and $-1 \in H$ then $S=\left(D_{1} \sqcap R_{0}[H / B(H)]\right)[G(R) / H]$, and $G\left(R_{0}\right) \subset H$.

(d) If $H \not \subset G\left(R_{0}\right)$ and $-1 \notin H$ then $S=\left(\mathbb{Z} \sqcap R_{0}[H / B(H)]\right)[G(R) / \pm H]$ and $G\left(R_{0}\right) \subset \pm H$.

Proof. If $H=1$ then $S=R\left[E_{1}\right]$ by (1.1), which gives (a). So assume $|H|>1$. Further suppose that $H \subset G\left(R_{0}\right)$ so that $B(H)=H \cap B(R)=$ $H \cap G\left(R_{0}\right)=H$. Then $-H^{\cdot} \subset T \subset G\left(R_{0}\right)$. Hence $\pm H \subset \pm T \subset G\left(R_{0}\right)$. Thus if (1.3)(a) holds, so that $G\left(R_{0}\right)=B(R)= \pm B(H)= \pm H$, then $B(R)= \pm T$ also. So we are always in case (b) of (1.3). Then, since $G(S) / B(S) \cong$ $G(R) / B(R)$, we have $S=S_{0}\left[E_{n}\right]$, where $S_{0}=W(B(S))$. From $H \subset B(R)$ we have that $S_{0}$ is an $H$-extension of $R_{0}$.

Next suppose that $H \not \subset G\left(R_{0}\right)$. We still have that $G\left(R_{0}\right)=B(R)$. If $B(R)= \pm T$ then $H \subset B(R)$, contrary to our assumption. Thus we are in Case (a) of (1.3). First say that $-1 \in H$. Note that $-1 \in H \cap B(R)=B(H)$ also. Then $B(R)=B(H)$ and $B(S)=\{1, \alpha\} H$. Thus $S=S_{0}[G(R) / H]$, for $S_{0}=W(\{1, \alpha\} H)$, since $G(S) / B(S) \cong G(R) / H$.

Now $D_{S_{0}}\langle 1,-\alpha\rangle=D_{S}\langle 1,-a\rangle \cap\{1, \alpha\} H=\{1, \alpha\} H$. Hence $\alpha \in \operatorname{rad}\left(S_{0}\right)$. Write $S_{0}=D_{1} \sqcap S_{1}$, for some Witt ring $S_{1}$ and with $D_{1}$, generated by $\alpha$, being $\mathbb{Z}_{2}\left[E_{1}\right]$ or $\mathbb{Z}_{4}$ using [4, p. 104]. Note that $S_{1}=W(H)$.

If $h \in H \backslash B(H)$ then $D_{R}\langle 1,-h\rangle=\{1,-h\}$ and $D_{S}\langle 1,-h\rangle=\{1, \alpha,-h$, $-\alpha h\}$ so that $D_{S_{1}}\langle 1,-h\rangle=D_{S}\langle 1,-h\rangle \cap H=\{1,-h\}$. Similarly, $D_{S_{1}}\langle h\rangle=$ $\{1, h\}$. And if $h \in B(H)$ then $D_{S_{1}}\langle 1,-h\rangle=\{1, \alpha\} D_{R}\langle 1,-h\rangle \cap H$ $=D_{R}\langle 1,-h\rangle \cap H=D_{R}\langle 1,-h\rangle \cap B(H)=D_{R_{0}}\langle 1,-h\rangle$. Thus $S_{1}=R_{0}[H / B(H)]$.

We still suppose $H \not \subset G\left(R_{0}\right)$, so that we are in Case (a) of (1.3), and now say that $-1 \notin H$. Then $S=S_{0}[G(R) / \pm H]$ as $G(S) / B(S)=\{1, \alpha\} G(R) / \pm$ $\{1, \alpha\} H \cong G(R) / \pm H$. Here $S_{0}=W( \pm\{1, \alpha\} H)$.

Now $D_{S_{0}}\langle 1,-\alpha\rangle=\{1, \alpha\} H$ has index two in $G\left(S_{0}\right)= \pm\{1, \alpha\} H$. Further $\alpha \notin D_{S}\langle 1,-\alpha\rangle$ else $-1 \in\{1,-\alpha\} H$ and $-1 \in H$. Thus we have an orthogonal decomposition in the sense of [1] :

$$
G\left(S_{0}\right)=\{1, \alpha\} \perp D_{S}\langle 1,-\alpha\rangle .
$$

Set $S_{1}=W(\{1, \alpha\})$ and $S_{2}=W\left(D_{S}\langle 1,-\alpha\rangle\right)$. Note that $S_{1}=\mathbb{Z}$ as $-1 \notin$ $D_{S}\langle 1,-\alpha\rangle$. If $h \in \pm H \backslash \pm B(H)$ then $D_{R}\langle 1, \pm h\rangle=\{1, \pm h\}, D_{S}\langle 1,-h\rangle=$ $\{1, \alpha,-h,-\alpha h\}$ and $D_{S}\langle 1, h\rangle=\{1, h\}$. Thus we have $D_{S_{1}}\langle 1, \pm h\rangle=$ $D_{S}\langle 1, \pm h\rangle \cap \pm H=\{1, \pm h\}$. So $S_{2}=S_{3}[H / B(H)]$, for some Witt ring $S_{3}$. $S_{2}$ is indeed a group ring as $H \not \subset G\left(R_{0}\right)$ implies $H \neq B(H)$.

We wish to apply $[\mathbf{1}, 3.4]$ and deduce that the decomposition (1.6) yields a product of Witt rings. First we need to handle the case where $S_{2}$ is decomposable, that is, $S_{2}=\mathbb{Z}\left[E_{1}\right]$. In this case $\left|G\left(S_{2}\right)\right|=\left|D_{S}\langle 1,-\alpha\rangle\right|=4$ so that $H=\{1, t\}$, for some $t \notin G\left(R_{0}\right)$ and $G\left(R_{0}\right)=\{ \pm 1\}$ as $G\left(R_{0}\right) \subset$ $\pm H$. Now $D_{S}\langle 1,-t\rangle=\{1, \alpha,-t,-\alpha t\}$ so we consider instead the orthogonal decomposition:

$$
\{1, t\} \perp D_{S}\langle 1,-t\rangle \text {. }
$$


Now $Q_{S}(\{1, t\})=\{1, q(t,-1)\}$ and $Q_{S}\left(D_{S}\langle 1,-t\rangle\right)=\{1, q(\alpha,-1), q(-t,-1)$, $q(-\alpha t,-1)\}$, using $q(\alpha,-t)=q(\alpha,-1)$ and $q(t,-\alpha t)=1$. Then $Q_{S}(\{1, t\}) \cap$ $Q_{S}\left(D_{S}\langle 1,-t\rangle\right)=1$. Thus:

$$
S_{0}=W(\{1, t\}) \sqcap W\left(D_{S}\langle 1,-t\rangle\right) .
$$

Now $W(\{1, t\})=\mathbb{Z}$ as $-1 \notin D_{S}\langle 1,-t\rangle$ and $W\left(D_{S}\langle 1,-t\rangle\right)=R_{0}[\{1, \alpha\}]$ since $\alpha$ is birigid in qf st and $G\left(R_{0}\right)=\{ \pm 1\}$. This gives the desired result of (d).

We now apply $[\mathbf{1}, 3.4]$ and obtain that either (1.6) yoilds a product or $Q_{S}(\{1, \alpha\})=Q_{S}\left(D_{S}\langle 1,-\alpha\rangle\right)$. But if $t \in H \backslash G\left(R_{0}\right)$ then $q(t, t)=q(t,-1)$ and $q(\alpha, \alpha)=q(\alpha,-1)$ are distinct, since $-1 \notin D_{S}\langle 1,-\alpha t\rangle$. Hence the decomposition (1.6) in fact yields the product $S_{0}=S_{1} \sqcap S_{2}$. We have already seen that $S_{1}=\mathbb{Z}$ and that $S_{2}=S_{3}[H / B(H)]$. If $h \in \pm B(H)$ then $D_{S_{3}}\langle 1,-h\rangle=D_{S_{1}}\langle 1,-h\rangle=D_{R}\langle 1,-h\rangle=D_{R_{0}}\langle 1,-h\rangle$. So $S_{3}=R_{0}$.

$H$-extensions are motivated by the behavior of odd degree field extensions. (1.5) and other lemmas do mimic the results expected in the field case, at least for valued fields. Pointing out these parallels is speculation (there may be no field extensions yielding an $H$-extension) but it is instructive.

Suppose then that $K / F$ is an odd degree extension with $W K$ an $H$ extension of $W F$. Further suppose that $K$ has a 2-henselian valuation $B$ with a basic residue field $k_{B}$. If $\alpha$ is not a unit, modulo squares, then $\alpha$ is birigid and we have case (a) of (1.5). Otherwise, $\alpha$ pushes down to $\bar{\alpha} \in k_{B}$. Set $A=B \cap F$. If $A$ has a basic residue field $k_{A}$ then $W k_{A} \subset W k_{B}$, $\bar{H}=D\langle 1,-\bar{\alpha}\rangle \cap k_{B}$ and $W k_{B}$ is an $\bar{H}$-extension of $W k_{A}$. This is (1.5)(b). If $k_{A}$ is not basic then $H$ contains birigid elements of $F$ and so $B\left(k_{A}\right) \subset \pm H$. This yields cases (c) and (d) of (1.5).

\section{Local type rings.}

Notation. For a subset $A \subset G(R)$ set:

$$
C_{R}(A)=\bigcap_{a \in A} D_{R}\langle 1,-a\rangle .
$$

Lemma 2.1. Suppose that $S$ is an $H$-extension of $R$. Suppose $k \in C_{R}(H) \backslash$ H. Then $Q_{R}(H) \cap Q_{R}(k)=1$.

Proof. Let $\rho \in Q_{R}(H) \cap Q_{R}(k)$ so that $\rho=q(h, x)=q(k, y)$ with $h \in H$, and $x, y \in G(R)$. Since $H \subset D_{S}\langle 1,-\alpha\rangle$ we have that $q(k, y)=q(\alpha x, h)$. By linkage there exists a $t \in G(S)$ such that:

$$
q(k, y)=q(k, t)=q(\alpha x, t)=q(\alpha x, h) .
$$

The first equality gives $t y \in D_{S}\langle 1,-k\rangle=D_{R}\langle 1,-k\rangle \subset G(R)$, since $k \notin H$. Hence $t \in G(R)$. The second equality gives:

$$
t \in D_{S}\langle 1,-\alpha x k\rangle \cap G(R)=D_{R}\langle 1,-x k\rangle \cap H .
$$


This implies $t \in D_{R}\langle 1,-x\rangle$ since $H \subset D_{R}\langle 1,-k\rangle$. The third equality gives:

$$
h t \in D_{S}\langle 1,-\alpha x\rangle \cap G(R)=D_{R}\langle 1,-x\rangle \cap H .
$$

Hence $h \in D_{R}\langle 1,-x\rangle$ and $\rho=q(x, h)=1$.

The small Witt rings of local type will often be treated separately. The only local type Witt ring with two generators is $\mathbb{Z}$. There are two Witt rings of local type on four generators and both are group rings. If $L$ is local type and $|G(L)| \geq 8$ then $L$ is not a group ring. See [4, Chapter 5, Section 3] for details.

Lemma 2.2. Suppose $R=L \sqcap R_{2}$, with $L$ a Witt ring of local type and $|G(L)| \geq 8$. Let $\pi_{1}$ be the projection map of $G(R)$ onto $G(L)$. Let $S$ be an $H$-extension of $R$. Then $\pi_{1}(H)=1$ or $G(L)$.

Proof. Set $B=\pi_{1}(H)$ and write $Q(L)=\{1, \rho\}$. Suppose that $B \neq 1$. If $(u, v) \in H$ with $u \neq 1$ then pick $r \in G(L) \backslash D_{L}\langle 1,-u\rangle$. We get $q((u, v),(r, 1))$ $=(\rho, 1)$ and so $(\rho, 1) \in Q(H)$.

Now $H \subset B \times G\left(R_{2}\right)$ so that $C_{L}(B) \times 1=C_{R}\left(B \times G\left(R_{2}\right)\right) \subset C_{R}(H)$. If $C_{L}(B)=1$ then $B=G(L)$ and we are done. So suppose there exists $1 \neq$ $z \in C_{L}(B)$. Set $h=(z, 1) \in C_{R}(H)$. Then $Q(h)=\{1,(\rho, 1)\} \subset Q(H)$. By (2.1) we must have $h \in H$ and so $C_{L}(B) \times 1 \subset H$. In particular, $C_{L}(B) \subset B$.

Continue to let $h=(z, 1)$ where $1 \neq z \in C_{L}(B)$. We claim there exist an $h_{1} \in H$ and an $x \in D_{R}\langle 1,-h\rangle$ such that $q\left(x, h_{1}\right)=(\rho, 1)$. Suppose not. We consider any $x=(a, 1)$ with $a \in D_{L}\langle 1,-z\rangle$. Then $q(x,(u, v))=(\rho, 1)$ unless $a \in D_{L}\langle 1,-u\rangle$. Thus $D_{L}\langle 1,-z\rangle \subset D_{L}\langle 1,-u\rangle$ for all $(u, v) \in H$, that is, $D_{L}\langle 1,-z\rangle \subset C_{L}(B)$. Then $C_{L}(B)=D_{L}\langle 1,-z\rangle$ and so $B=\{1, z\}$. But $C_{L}(B) \subset B$ so that $D_{L}\langle 1,-z\rangle \subset\{1, z\}$ and $|G(L)|=4$, a case we are excluding.

Thus there does exist an $h_{1} \in H$ and an $x \in D_{R}\langle 1,-h\rangle$ such that $q\left(x, h_{1}\right)=(\rho, 1)$. Then in $S$ we have $q\left(\alpha x, h_{1}\right)=(\rho, 1)$ and so $Q_{S}(h) \subset$ $Q_{S}(\alpha x)$. We obtain:

$$
\left|Q_{S}(\alpha x) \cap Q_{S}(h)\right|=2 .
$$

On the other hand:

$$
D_{S}\langle 1,-\alpha x\rangle \cap D_{S}\langle 1,-h\rangle=\{1,-\alpha x\}\left(D_{R}\langle 1,-x\rangle \cap H\right) \cap\{1, \alpha\} D_{R}\langle 1,-h\rangle .
$$

Here $D_{R}\langle 1,-x\rangle \cap H \subset D_{R}\langle 1,-h\rangle$ as $h \in C_{R}(H)$. Also, by construction, $x$ is an element of $D_{R}\langle 1,-h\rangle$ and $h \in C_{R}(H) \subset D_{R}\langle 1,-h\rangle$. So $-1, x \in$ $D_{R}\langle 1,-h\rangle$ and $-\alpha x \in \alpha D_{R}\langle 1,-h\rangle$. Thus $D_{S}\langle 1,-\alpha x\rangle \subset D_{S}\langle 1,-h\rangle$. By [4, $5.2]$ :

$$
\left|Q_{S}(\alpha x) \cap Q_{S}(h)\right|=\frac{\left|D_{S}\langle 1,-\alpha x h\rangle\right|}{\left|D_{S}\langle 1,-\alpha x\rangle \cap D_{S}\langle 1,-h\rangle\right|}=\frac{2\left|D_{R}\langle 1,-x h\rangle \cap H\right|}{2\left|D_{R}\langle 1,-x\rangle \cap H\right|},
$$

and $D_{R}\langle 1,-x h\rangle \cap H=D_{R}\langle 1,-x\rangle \cap H$ as $h \in C_{R}(H)$ implies $H \subset D_{R}\langle 1,-h\rangle$. So $\left|Q_{S}(\alpha x) \cap Q_{S}(h)\right|=1$, a contradiction. Hence $\pi_{1}(H)=G(L)$. 
Corollary 2.3. Suppose $R$ is of local type with $|G(R)| \neq 4$. If $S$ is an $H$ extension of $R$ then either:

(a) $H=1$ and $S=R\left[E_{1}\right]$, with $E_{1}$ generated by $\alpha$, or

(b) $H=G(R)$ and $S=D_{1} \sqcap R$, with $D_{1}$ generated by $\alpha$.

Proof. If $|G(R)|=2$ then it is clear that $H=1$ or $H=G(R)$. If $|G(R)| \geq 8$ the take $R_{2}=1$ in (2.2) to get $H=1$ or $G(R)$. Now apply (1.1).

\section{Products: General Lemmas.}

We start with a lemma that may be of general interest.

Lemma 3.1. Let $R$ be a Witt ring of elementary type. Let $K$ be a proper subgroup of $G(R)$ and let $y \in G(R)$. If

$$
G(R)=\bigcup_{k \in K} D\langle 1,-y k\rangle
$$

then $y \in \operatorname{rad}(R) \cdot K$.

Proof. We first prove the result for non-degenerate $R$ where we must show $y \in K$. It suffices to prove this for subgroups $K$ of index two. Namely, if $K_{0}$ is any subgroup satisfying the hypothesis let $\mathcal{A}$ denote the set of subgroups $K$ of index two that contain $K_{0}$. Then for any $K \in \mathcal{A}$ :

$$
G(R)=\bigcup_{k \in K_{0}} D\langle 1,-y k\rangle \subset \bigcup_{k \in K} D\langle 1,-y k\rangle .
$$

Assuming the result holds for subgroups of index two, we obtain $y \in K$. Then $y \in \bigcap_{K \in \mathcal{A}} K=K_{0}$, as desired.

So suppose $[G(R): K]=2$. We work by induction on $|G(R)|$. We need to prove that $y \in K$ when $R$ is of local type, a group ring or a product. First suppose $R$ is of local type. Then $K=D\langle 1,-a\rangle$, for some $a \in G(R)$. We have:

$$
G(R)=\bigcup_{k \in D\langle 1,-a\rangle} D\langle 1,-y k\rangle=D\langle 1,-y, a y\rangle .
$$

Multiplying by $-a$ gives $G(R)=D\langle\langle-a,-y\rangle\rangle^{\prime}$, the pure part of the Pfister form $\langle\langle-a,-y\rangle\rangle$. In particular, $-1 \in D\langle\langle-a,-y\rangle\rangle^{\prime}$ so $\langle\langle-a,-y\rangle\rangle=0$ and $y \in D\langle 1,-a\rangle=K$.

Next let $R=R_{0}\left[E_{1}\right]$, with $E_{1}=\{1, t\}$ and $\left|G\left(R_{0}\right)\right| \geq 2$ (if $G\left(R_{0}\right)=1$ then $R$ is degenerate). Suppose $y \notin K$. We claim $G\left(R_{0}\right) \subset K$. Choose any $g \in G\left(R_{0}\right)$. Then there exist $k_{1}, k_{2} \in K$ with $-g t \in D\left\langle 1,-y k_{1}\right\rangle$ and $-t \in$ $D\left\langle 1,-y k_{2}\right\rangle$. We get $g t=y k_{1}$ and $t=y k_{2}$ since $y \notin K$. Hence $g=k_{1} k_{2} \in K$. This proves the claim. Both $G\left(R_{0}\right)$ and $K$ have index two so $K=G\left(R_{0}\right)$. From $t=y k_{2}$ we have $y \in t G\left(R_{0}\right)$. Pick $g \in G\left(R_{0}\right)$. Then $g \in D\left\langle 1,-y k_{3}\right\rangle$ for some $k_{3} \in K$. But $y k_{3} \in y K=t G\left(R_{0}\right)$, so this is impossible. The contradiction implies $y \in K$. 
Lastly, say $R=R_{1} \sqcap R_{2}$. Write $y=\left(y_{1}, y_{2}\right)$. Now $K \cap\left(G\left(R_{1}\right) \times 1\right)$ is a subgroup of index at most two in $G\left(R_{1}\right) \times 1$. Let $K_{1}$ be its projection into $G\left(R_{1}\right)$. Similarly, let $K_{2}$ be the projection of $K \cap\left(1 \times G\left(R_{2}\right)\right)$ into $G\left(R_{2}\right)$. Then $\left[G\left(R_{i}\right): K_{i}\right] \leq 2$, for $i=1,2$. If $K_{2}=G\left(R_{2}\right)$ then:

$$
\bigcup_{k \in K} D_{R}\langle 1,-y k\rangle=\bigcup_{k_{1} \in K_{1}} D\left\langle 1,-y_{1} k_{1}\right\rangle \times G\left(R_{2}\right)
$$

so that $G\left(R_{1}\right)=\bigcup_{K_{1}} D\left\langle 1,-y_{1} k_{1}\right\rangle$. By induction then $y_{1} \in K_{1}$ and hence $y=\left(y_{1}, y_{2}\right) \in K_{1} \times G\left(R_{2}\right)=K$. In the same way, if $K_{1}=G\left(R_{1}\right)$ then $y \in K$ as desired. So we may assume that $\left[G\left(R_{i}\right): K_{i}\right]=2$ for $i=1,2$. Write $K=\{1, \gamma\} K_{1} \times K_{2}$. We have:

$$
G(R)=\bigcup_{k_{1} \in K_{1}, k_{2} \in K_{2}}\left(D\left\langle 1,-y_{1} k_{1}\right\rangle \times D\left\langle 1,-y_{2} k_{2}\right\rangle\right.
$$

$$
\left.\cup D\left\langle 1,-y_{1} \gamma_{1} k_{1}\right\rangle \times D\left\langle 1,-y_{2} \gamma_{2} k_{2}\right\rangle\right),
$$

where $\gamma=\left(\gamma_{1}, \gamma_{2}\right)$.

Suppose $y_{1} \in K_{1}$. If $G\left(R_{1}\right)=\cup D\left\langle 1,-y_{1} \gamma_{1} k_{1}\right\rangle$ then by induction we have $y_{1} \gamma_{1} \in K_{1}$ and so $\gamma_{1} \in K_{1}$. Then $K=K_{1} \times G\left(R_{2}\right)$ and $K_{2}=G\left(R_{2}\right)$ a case we have already covered. We may thus assume there exists a $g_{1} \in$ $G\left(R_{1}\right) \backslash \cup D\left\langle 1,-y_{1} \gamma_{1} k_{1}\right\rangle$. Then $g_{1} \times G\left(R_{2}\right) \subset \cup\left(D\left\langle 1,-y_{1} k_{1}\right\rangle \times D\left\langle 1,-y_{2} k_{2}\right\rangle\right)$ and so $G\left(R_{2}\right)=\cup D\left\langle 1,-y_{2} k_{2}\right\rangle$. By induction $y_{2} \in K_{2}$ and $y=\left(y_{1}, y_{2}\right) \in$ $K_{1} \times K_{2} \subset K$, and we are done.

We may thus assume $y_{1} \notin K_{1}$. Similarly, $y_{2} \notin K_{2}$. Pick, for $i=1$, 2, a $g_{i} \in G\left(R_{i}\right) \backslash \cup D\left\langle 1,-y_{i} k_{i}\right\rangle$, which is possible by induction. Then $g_{1} \times G\left(R_{2}\right) \subset$ $\cup\left(D\left\langle 1,-y_{1} \gamma_{1} k_{1}\right\rangle \times D\left\langle 1,-y_{2} \gamma_{2} k_{2}\right\rangle\right)$ and so $G\left(R_{2}\right)=\cup D\left\langle 1,-y_{2} \gamma_{2} k_{2}\right\rangle$. By induction once more, we have $y_{2} \gamma_{2} \in K_{2}$. Similarly, $y_{1} \gamma_{1} \in K_{1}$. Then $y \in\left(\gamma_{1}, \gamma_{2}\right)\left(K_{1} \times K_{2}\right) \subset K$, as desired. This proves the result for nondegenerate $R$.

Now suppose $R$ is degenerate. Write $R=D \sqcap R_{2}$, with $\operatorname{rad}(R)=G(D) \times 1$ and $R_{2}$ non-degenerate. Let $\pi_{2}$ be the projection of $G(R)$ onto $G\left(R_{2}\right)$. Set $K_{2}=\pi_{2}(K)$ and write $y=\left(y_{1}, y_{2}\right)$, with $y_{1} \in G(D)$ and $y_{2} \in G\left(R_{2}\right)$. Our assumption is:

$$
\begin{aligned}
G(R)=G(D) \times G\left(R_{2}\right) & =\bigcup_{\left(k_{1}, k_{2}\right) \in K} D\left\langle(1,1),-\left(y_{1} k_{1}, y_{2} k_{2}\right)\right\rangle \\
& =G(D) \times\left(\bigcup_{k_{2} \in K_{2}} D_{R_{2}}\left\langle 1,-y_{2} k_{2}\right\rangle\right) .
\end{aligned}
$$

From the non-degenerate case we get $y_{2} \in K_{2}=\pi_{2}(K)$. Thus there exists a $d \in G(D)$ such that $\left(d, y_{2}\right) \in K$. Hence $y=\left(y_{1}, y_{2}\right)=\left(d y_{1}, 1\right)\left(d, y_{2}\right) \in$ $\operatorname{rad}(R) \cdot K$.

Our key reduction lemma follows. 
Lemma 3.2. Let $R=R_{1} \sqcap R_{2}$ and suppose $S$ is an $H$-extension of $R$. If $H=H_{1} \times G\left(R_{2}\right)$ then there exists a Witt ring $T$ that is an $H_{1}$-extension of $R_{1}$ such that $S \cong T \sqcap R_{2}$.

Proof. We first construct $T$. Let $G(T)$ be a group containing $G\left(R_{1}\right)$ as a subgroup of index 2 ; write $G(T)=\{1, \beta\} G\left(R_{1}\right)$. Let $\varphi: G(T) \rightarrow$ $\{1, \alpha\}\left(G\left(R_{1}\right) \times 1\right)$ be the isomorphism sending $g_{1} \mapsto\left(g_{1}, 1\right)$ and $\beta g_{1} \mapsto$ $\alpha\left(g_{1}, 1\right)$, where $g_{1} \in G\left(R_{1}\right)$. For $z \in G(T)$ define:

$$
D_{T}\langle 1,-z\rangle=\varphi^{-1}\left(D_{S}\langle 1,-\varphi(z)\rangle \cap\{1, \alpha\}\left(G\left(R_{1}\right) \times 1\right)\right) .
$$

We check that $T$ is an $H_{1}$-extension of $R_{1}$. If $z \in G\left(R_{1}\right) \backslash H_{1}$ then:

$$
\begin{aligned}
D_{T}\langle 1,-z\rangle & =\varphi^{-1}\left(\left(D_{R_{1}}\langle 1,-z\rangle \times G\left(R_{2}\right)\right) \cap\{1, \alpha\}\left(G\left(R_{1}\right) \times 1\right)\right) \\
& =\varphi^{-1}\left(D_{R_{1}}\langle 1,-z\rangle \times 1\right) \\
& =D_{R_{1}}\langle 1,-z\rangle .
\end{aligned}
$$

If $z \in H_{1}$ then:

$$
\begin{aligned}
D_{T}\langle 1,-z\rangle & =\varphi^{-1}\left(\{1, \alpha\}\left(D_{R_{1}}\langle 1,-z\rangle \times G\left(R_{2}\right)\right) \cap\{1, \alpha\}\left(G\left(R_{1}\right) \times 1\right)\right) \\
& =\varphi^{-1}\left(\{1, \alpha\}\left(D_{R_{1}}\langle 1,-z\rangle \times 1\right)\right) \\
& =\{1, \beta\} D_{R_{1}}\langle 1,-z\rangle .
\end{aligned}
$$

Lastly, if $z \in G\left(R_{1}\right)$ then:

$$
D_{T}\langle 1,-\beta z\rangle=\varphi^{-1}\left(D_{S}\langle 1,-\alpha(z, 1)\rangle \cap\{1, \alpha\}\left(G\left(R_{1}\right) \times 1\right)\right) .
$$

Now:

$$
\begin{aligned}
D_{S}\langle 1,-\alpha(z, 1)\rangle & =\{1,-\alpha(z, 1)\}\left(\left(D_{R_{1}}\langle 1,-z\rangle \times G\left(R_{2}\right)\right) \cap\left(H_{1} \times G\left(R_{2}\right)\right)\right) \\
& =\{1,-\alpha(z, 1)\}\left(\left(D_{R_{1}}\langle 1,-z\rangle \cap H_{1}\right) \times G\left(R_{2}\right)\right) .
\end{aligned}
$$

Since $(1,-1) \in D_{R_{1}}\langle 1,-z\rangle \cap H_{1} \times G\left(R_{2}\right)$ we have:

$$
D_{S}\langle 1,-\alpha(z, 1)\rangle=\{1, \alpha(-z, 1)\}\left(\left(D_{R_{1}}\langle 1,-z\rangle \cap H_{1}\right) \times G\left(R_{2}\right)\right) .
$$

Hence:

$$
\begin{aligned}
D_{T}\langle 1,-\beta z\rangle= & \varphi^{-1}\left(\{1, \alpha(-z, 1)\}\left(D_{R_{1}}\langle 1,-z\rangle \cap H_{1}\right) \times G\left(R_{2}\right)\right) \\
& \cap\{1, \alpha(-z, 1)\}\left(G\left(R_{1}\right) \times 1\right) \\
= & \varphi^{-1}\left(\{1, \alpha(-z, 1)\}\left(D_{R_{1}}\langle 1,-z\rangle \cap H_{1}\right) \times 1\right) \\
= & \{1,-\beta z\}\left(D_{R_{1}}\langle 1,-z\rangle \cap H_{1}\right) .
\end{aligned}
$$

We begin the verification that $\left(G(T), D_{T}\right)$ is linked, so that $T$ is indeed a Witt ring. Let $t=\alpha^{\epsilon_{1}}(u, v) \in G(S)$ and let $\beta^{\epsilon_{2}} x, \beta^{\epsilon_{3}} y \in G(T)$ with each $\epsilon_{i}=0$ or 1 .

Claim. If $t \in \varphi\left(\beta^{\epsilon_{3}} y\right) D_{S}\left\langle 1,-\varphi\left(\beta^{\epsilon_{2}} x\right)\right\rangle$ then $\beta^{\epsilon_{1}} u \in \beta^{\epsilon_{3}} y D_{T}\left\langle 1,-\beta^{\epsilon_{2}} x\right\rangle$.

We first assume that $\epsilon_{1}=0$. We have four cases: 
Case 1. $\epsilon_{2}=0, \epsilon_{3}=0$. Here $(u y, v) \in D_{S}\langle 1,-(x, 1)\rangle$, hence $u y \in D_{R_{1}}\langle 1,-x\rangle$ $\subset D_{T}\langle 1,-x\rangle$.

Case 2. $\epsilon_{2}=0, \epsilon_{3}=1$. Here $\alpha(u y, v) \in D_{S}\langle 1,-(x, 1)\rangle$. We must have that $x \in H_{1}$ so $\alpha(u y, v) \in\{1, \alpha\}\left(D_{R_{1}}\langle 1,-x\rangle \times G\left(R_{2}\right)\right)$. Then $u y \in D_{R_{1}}\langle 1,-x\rangle$. Thus $\beta$ uy $\in D_{T}\langle 1,-x\rangle=\{1, \beta\} D_{R_{1}}\langle 1,-x\rangle$.

Case 3. $\epsilon_{2}=1, \epsilon_{3}=0$. Here:

$$
\begin{aligned}
(u y, v) & \in\{1,-\alpha(x, 1)\}\left(\left(D_{R_{1}}\langle 1,-x\rangle \times G\left(R_{2}\right)\right) \cap H\right) \\
& =\{1,-\alpha(x, 1)\}\left(\left(D_{R_{1}}\langle 1,-x\rangle \cap H_{1}\right) \times G\left(R_{2}\right)\right) .
\end{aligned}
$$

Thus $u y \in D_{R_{1}}\langle 1,-x\rangle \cap H_{1}$. We obtain

$$
u y \in D_{T}\langle 1,-\beta x\rangle=\{1,-\beta x\}\left(D_{R_{1}}\langle 1,-x\rangle \cap H_{1}\right) .
$$

Case 4. $\epsilon_{2}=1, \epsilon_{3}=1$. Here $\alpha(u y, v) \in\{1,-\alpha(x, 1)\}\left(\left(D_{R_{1}}\langle 1,-x\rangle \cap H_{1}\right) \times\right.$ $\left.G\left(R_{2}\right)\right)$ so that $(-x u y, v) \in\left(D_{R_{1}}\langle 1,-x\rangle \cap H_{1}\right) \times G\left(R_{2}\right)$. Thus $-x u y \in$ $D_{R_{1}}\langle 1,-x\rangle \cap H_{1}$ and $\beta u y \in D_{T}\langle 1,-\beta x\rangle=\{1,-\beta x\}\left(D_{R_{1}}\langle 1,-x\rangle \cap H_{1}\right)$.

The four cases with $\epsilon_{1}=1$ are identical to the four above cases. For example, if $\epsilon_{1}=1, \epsilon_{2}=0, \epsilon_{3}=0$ then we have $\alpha(u y, v) \in D_{S}\langle 1,-(x, 1)\rangle$, which is Case 2 above. Thus the Claim is proven.

We can now check linkage in $T$. Let $x, y, z, w \in G(T)$ and suppose:

$$
x D_{T}\langle 1,-y\rangle \cap D_{T}\langle 1,-y z\rangle \cap w D_{T}\langle 1,-z\rangle \neq \emptyset .
$$

Apply $\varphi$ to get:

$$
\varphi(x) D_{S}\langle 1,-\varphi(y)\rangle \cap D_{S}\langle 1,-\varphi(y z)\rangle \cap \varphi(w) D_{S}\langle 1,-\varphi(z)\rangle \neq \emptyset .
$$

By linkage on $S$, there exists a $t \in G(S)$ in:

$$
\varphi(y) D_{S}\langle 1,-\varphi(x)\rangle \cap D_{S}\langle 1,-\varphi(x w)\rangle \cap \varphi(z) D_{S}\langle 1,-\varphi(w)\rangle .
$$

Now apply the

\section{Claim.}

$$
y D_{T}\langle 1,-x\rangle \cap D_{T}\langle 1,-x w\rangle \cap z D_{T}\langle 1,-w\rangle \neq \emptyset,
$$

as desired.

Lastly, set $W=T \sqcap R_{2}$. Then $G(W)=\left(\{1, \beta\} G\left(R_{1}\right)\right) \times G\left(R_{2}\right)$. Set $\gamma=(\beta, 1)$ so that $G(W)=\{1, \gamma\}\left(G\left(R_{1}\right) \times G\left(R_{2}\right)\right)=\{1, \gamma\} G(R)$. We will show $W$ is an $H$-extension of $R$, via $\gamma$, and hence that $S \cong W$.

First let $h=\left(h_{1}, g_{2}\right) \in H$, where $h_{1} \in H_{1} \subset G\left(R_{1}\right)$ and $g_{2} \in G\left(R_{2}\right)$. Then:

$$
\begin{aligned}
D_{W}\langle 1,-h\rangle & =D_{T}\left\langle 1,-h_{1}\right\rangle \times D_{R_{2}}\left\langle 1,-g_{2}\right\rangle \\
& =\left(\{1, \beta\} D_{R_{1}}\left\langle 1,-h_{1}\right\rangle\right) \times D_{R_{2}}\left\langle 1,-g_{2}\right\rangle \\
& =\{1, \gamma\} D_{R}\langle 1,-h\rangle .
\end{aligned}
$$


Next let $g=\left(g_{1}, g_{2}\right) \in G(R) \backslash H$, with $g_{1} \in G\left(R_{1}\right) \backslash H_{1}$ and $g_{2} \in G\left(R_{2}\right)$. Then:

$$
\begin{aligned}
D_{W}\langle 1,-g\rangle & =D_{T}\left\langle 1,-g_{1}\right\rangle \times D_{R_{2}}\left\langle 1,-g_{2}\right\rangle \\
& =D_{R_{1}}\left\langle 1,-g_{1}\right\rangle \times D_{R_{2}}\left\langle 1,-g_{2}\right\rangle=D_{R}\langle 1,-g\rangle .
\end{aligned}
$$

Lastly, let $g=\left(g_{1}, g_{2}\right) \in G(R)$, with $g_{1} \in G\left(R_{1}\right)$ and $g_{2} \in G\left(R_{2}\right)$. Then:

$$
\begin{aligned}
D_{W}\langle 1,-\gamma g\rangle= & D_{W}\left\langle 1,-\left(\beta g_{1}, g_{2}\right)\right\rangle \\
= & D_{R_{1}}\left\langle 1,-\beta g_{1}\right\rangle \times D_{R_{2}}\left\langle 1,-g_{2}\right\rangle \\
= & \left(\left\{1,-\beta g_{1}\right\}\left(D_{R_{1}}\left\langle 1,-g_{1}\right\rangle \cap H_{1}\right)\right) \times D_{R_{2}}\left\langle 1,-g_{2}\right\rangle \\
= & \left(D_{R_{1}}\left\langle 1,-g_{1}\right\rangle \cap H_{1}\right) \times D_{R_{2}}\left\langle 1,-g_{2}\right\rangle \\
& \cup \gamma\left(-g_{1}\left(D_{R_{1}}\left\langle 1,-g_{1}\right\rangle \cap H_{1}\right) \times-g_{2} D_{R_{2}}\left\langle 1,-g_{2}\right\rangle\right) \\
= & \left\{1,-\gamma\left(g_{1}, g_{2}\right)\right\}\left(D_{R}\left\langle 1,-\left(g_{1}, g_{2}\right)\right\rangle \cap H\right) \\
= & \{1,-\gamma g\}\left(D_{R}\langle 1,-g\rangle \cap H\right) .
\end{aligned}
$$

Thus $W=T \sqcap R_{2}$ is an $H$-extension of $R$ and so is isomorphic to $S$.

Our last general lemma is the most technical, but it also does most of the work.

Lemma 3.3. Let $u \in G(R)$ and $h \in H$. Then:

$$
D_{R}\langle 1, u h,-h\rangle \backslash u H \subset \bigcup_{t \in h(D\langle 1,-u\rangle \cap H)} D_{R}\langle 1,-t\rangle .
$$

Proof. Let $w \in D_{R}\langle 1, u h,-h\rangle \backslash u H$. Then $w \in D_{R}\langle 1,-h v\rangle$ for some $v \in$ $D_{R}\langle 1,-u\rangle$ and $u w \notin H$. We have:

$$
q(\alpha u, h)=q(u, h)=q(u, v h)=q(u w, v h) .
$$

Thus, by linkage, there exists a $t \in G(S)$ such that:

$$
q(\alpha u, h)=q(\alpha u, t)=q(u w, t)=q(u w, v h) .
$$

The third equality gives $v h t \in D_{S}\langle 1,-u w\rangle$. Since $u w \notin H$ this implies $t \in G(R)$. Then the first two equalities give:

$$
\begin{aligned}
h t \in D_{S}\langle 1,-\alpha u\rangle \cap G(R) & =D_{R}\langle 1,-u\rangle \cap H, \\
t \in D_{S}\langle 1,-\alpha w\rangle \cap G(R) & =D_{R}\langle 1,-w\rangle \cap H .
\end{aligned}
$$

Hence $w \in D_{R}\langle 1,-t\rangle$ where $t \in h\left(D_{R}\langle 1,-u\rangle \cap H\right)$.

\section{Products: Degenerate Witt rings.}

If $R$ is a degenerate Witt ring then $R=D \sqcap R_{2}$, for some Witt ring $R_{2}$ and where $G(D)=\{1, d\}$, with $D_{D}\langle 1,1\rangle=D_{D}\langle 1, d\rangle=\{1, d\}$. We will often use the fact that if $(u, v) \in G(R)$ then $D_{R}\langle 1,-(u, v)\rangle=D_{R}\langle 1,-(d u, v)\rangle$. 
Lemma 4.1. Suppose $R=D \sqcap R_{2}$ is degenerate. Let $\pi_{1}$ be the projection of $G(R)$ onto $G(D)$. Let $S$ be an $H$-extension of $R$. Then either $G(D) \times 1 \subset H$ or $S$ is isomorphic to an $H_{0}$-extension of $R$, for some subgroup $H_{0} \subset G(R)$ with $\pi_{1}\left(H_{0}\right)=1$.

Proof. Suppose $(d, 1) \notin H$ and that $\pi_{1}(H) \neq 1$, so that $(d, y) \in H$ for some $y \in G\left(R_{2}\right)$. Let $G_{2}$ be the subgroup of $G\left(R_{2}\right)$ such that $1 \times G_{2}=$ $H \cap\left(1 \times G\left(R_{2}\right)\right)$. Then $H=\left(1 \times G_{2}\right) \cup\left(d \times y G_{2}\right)$. Set $H_{0}=1 \times\{1, y\} G_{2}$, and note that $\pi_{1}\left(H_{0}\right)=1$.

Let $\beta^{2}=1$ and set $G\left(S_{0}\right)=\{1, \beta\} G\left(R_{2}\right)$. Define $S_{0}$-value set s so that $S_{0}$ is an $H_{0}$-extension of $R$. We wish to show $S \cong S_{0}$. Extend $G_{2}$ to a subgroup $K$ of index two in $G\left(R_{2}\right)$ that does not contain $y$. Define $\varphi: G(S) \rightarrow G\left(S_{0}\right)$ by $\alpha \mapsto \beta$ and for $(u, v) \in G(R)$ :

$$
\varphi(u, v)= \begin{cases}(u, v), & \text { if } v \in K \\ (d u, v), & \text { if } v \notin K .\end{cases}
$$

It is quickly checked that $\varphi$ is an isomorphism. We will show:

$$
\varphi\left(D_{S}\langle 1,-s\rangle\right)=D_{S_{0}}\langle 1,-\varphi(s)\rangle,
$$

for all $s \in G(S)$. This shows both that $S_{0}$ is a Witt ring and that $S \cong S_{0}$.

Claim. If $(u, v) \in G(R)$ then $\varphi\left(D_{R}\langle 1,-(u, v)\rangle\right)=D_{R}\langle 1,-(u, v)\rangle$.

$$
\begin{aligned}
D_{R}\langle 1,-(u, v)\rangle= & \{1, d\} \times D_{R_{2}}\langle 1,-v\rangle \\
= & 1 \times\left(D_{R_{2}}\langle 1,-v\rangle \cap K\right) \cup d \times\left(D_{R_{2}}\langle 1,-v\rangle \cap K\right) \\
& \quad \cup 1 \times\left(D_{R_{2}}\langle 1,-v\rangle \cap y K\right) \cup d \times\left(D_{R_{2}}\langle 1,-v\rangle \cap y K\right) .
\end{aligned}
$$

Thus:

$$
\begin{aligned}
\varphi\left(D_{R}\langle 1,-(u, v)\rangle\right)= & 1 \times\left(D_{R_{2}}\langle 1,-v\rangle \cap K\right) \cup d \times\left(D_{R_{2}}\langle 1,-v\rangle \cap K\right) \\
& \cup d \times\left(D_{R_{2}}\langle 1,-v\rangle \cap y K\right) \cup 1 \times\left(D_{R_{2}}\langle 1,-v\rangle \cap y K\right) \\
= & D_{R}\langle 1,-(u, v)\rangle,
\end{aligned}
$$

proving the Claim.

We now check (4.2) in various cases. First suppose $s=(u, v) \in G(R)$, with $v \in K$. Then $s \in H$ iff $u=1$ and $v \in G_{2}$. We have $\varphi(s)=s$ and $\varphi(s) \in H_{0}$ iff $u=1$ and $v \in G_{2}$ iff $s \in H . D_{S}\langle 1,-s\rangle=\{1, \alpha\} D_{R}\langle 1,-s\rangle$ or $D_{R}\langle 1,-s\rangle$ depending on whether or not $s \in H$. So by the Claim, $\varphi\left(D_{S}\langle 1,-s\rangle\right)=$ $\{1, \beta\} D_{R}\langle 1,-s\rangle$ or $D_{R}\langle 1,-s\rangle$ depending on whether or not $\varphi(s) \in H_{0}$. Thus $\varphi\left(D_{S}\langle 1,-s\rangle\right)=D_{S_{0}}\langle 1,-\varphi(s)\rangle$.

Next suppose that $s=(u, v) \in G(R)$ with $v \in y K$. Then $s \in H$ iff $u=d$ and $v \in y G_{2}$. We have $\varphi(s)=(d u, v)$ so that $\varphi(s) \in H_{0}$ iff $u=d$ and $v \in y G_{2}$ iff $s \in H$. Again using the Claim:

$$
D_{S}\langle 1,-s\rangle= \begin{cases}\{1, \alpha\} D_{R}\langle 1,-s\rangle, & \text { if } s \in H \\ D_{R}\langle 1,-s\rangle, & \text { if } s \notin H .\end{cases}
$$


Thus:

$$
\varphi\left(D_{S}\langle 1,-s\rangle\right)= \begin{cases}\{1, \beta\} D_{R}\langle 1,-s\rangle, & \text { if } \varphi(s) \in H_{0} \\ D_{R}\langle 1,-s\rangle & \text { if } \varphi(s) \notin H_{0} .\end{cases}
$$

Now $D_{R}\langle 1,-s\rangle=D_{R}\langle 1,-(u, v)\rangle=D_{R}\langle 1,-(d u, v)\rangle=D_{R}\langle 1,-\varphi(s)\rangle$. Hence we have as desired that $\varphi\left(D_{S}\langle 1,-s\rangle\right)=D_{S_{0}}\langle 1,-\varphi(s)\rangle$.

Now suppose $s=\alpha(u, v) \in \alpha G(R)$. Then:

$$
\begin{aligned}
D_{S}\langle 1,-s\rangle & =\{1,-\alpha(u, v)\}\left(D_{R}\langle 1,-(u, v)\rangle \cap H\right) \\
& =\{1,-\alpha(u, v)\}\left[\left(\{1, d\} \times D_{R_{2}}\langle 1,-v\rangle\right) \cap\left(1 \times G_{2} \cup d \times y G_{2}\right)\right] \\
& =\{1,-\alpha(u, v)\}\left[1 \times\left(D_{R_{2}}\langle 1,-v\rangle \cap G_{2}\right) \cup d \times\left(D_{R_{2}}\langle 1,-v\rangle \cap y G_{2}\right)\right] .
\end{aligned}
$$

Now:

$$
\begin{aligned}
& \varphi\left(\left(1 \times\left(D_{R_{2}}\langle 1,-v\rangle \cap G_{2}\right)\right) \cup\left(d \times\left(D_{R_{2}}\langle 1,-v\rangle \cap y G_{2}\right)\right)\right) \\
& =1 \times\left(D_{R_{2}}\langle 1,-v\rangle \cap G_{2}\right) \cup 1 \times\left(D_{R_{2}}\langle 1,-v\rangle \cap y G_{2}\right) \\
& =1 \times\left(D_{R_{2}}\langle 1,-v\rangle \cap\{1, y\} G_{2}\right) \\
& =D_{R}\langle 1,-(u, v)\rangle \cap H_{0} .
\end{aligned}
$$

Thus if $v \in K$ then:

$$
\begin{aligned}
\varphi\left(D_{S}\langle 1,-s\rangle\right) & =\varphi\left(D_{S}\langle 1,-\alpha(u, v)\rangle\right) \\
& =\{1,-\beta(u, v)\}\left(D_{R}\langle 1,-(u, v)\rangle \cap H_{0}\right)=D_{S_{0}}\langle 1,-\varphi(s)\rangle,
\end{aligned}
$$

verifying (4.2) in this case.

Lastly, if $v \in y K$ then:

$$
\begin{aligned}
\varphi\left(D_{S}\langle 1,-s\rangle\right) & =\varphi\left(D_{S}\langle 1,-\alpha(u, v)\rangle\right) \\
& =\{1,-\beta(d u, v)\}\left(D_{R}\langle 1,-(u, v)\rangle \cap H_{0}\right) \\
& =\{1,-\beta(d u, v)\}\left(D_{R}\langle 1,-(d u, v)\rangle \cap H_{0}\right) \\
& =D_{S_{0}}\langle 1,-\varphi(s)\rangle .
\end{aligned}
$$

Thus (4.2) holds in all cases.

\section{Products: Local type factors.}

Lemma (3.3) looks simpler when one factor has local type.

Lemma 5.1. Suppose $R=L \sqcap R_{2}$, with $L$ of local type. Suppose $S$ is an $H$-extension of $R$. Let $h=\left(h_{1}, h_{2}\right) \in H$ and $u=\left(u_{1}, u_{2}\right) \in G(R)$ such that $u_{1} \notin D_{L}\left\langle 1,-h_{1}\right\rangle$ while $u_{2} \in D_{R_{2}}\left\langle 1,-h_{2}\right\rangle$. Then:

$$
\begin{aligned}
& G(R)=u H \cup\left(u_{1} \times G\left(R_{2}\right)\right) \cup \quad \bigcup \quad D_{R}\langle 1,-t\rangle . \\
& t \in h\left(D_{R}\langle 1,-u\rangle \cap H\right)
\end{aligned}
$$


Proof. Write $Q(L)=\{1, \rho\}$. Then $\langle\langle-u,-h\rangle\rangle=(\rho, 1)$. Hence $-D_{R}\langle\langle-u,-h\rangle\rangle^{\prime}=\{(x, y) \in G(R): x \neq 1\}$. Now $-u \cdot\langle-u,-h, u h\rangle \simeq$ $\langle 1, u h,-h\rangle$. Thus $D_{R}\langle 1, u h,-h\rangle=\left\{(x, y) \in G(R): x \neq u_{1}\right\}$. Apply (3.3).

Lemma 5.2. Suppose $R=L \sqcap R_{2}$, with $L$ of local type. Let $\pi_{1}$ be the projection of $G(R)$ onto $G(L)$. Let $S$ be an $H$-extension of $R$ and suppose that $\pi_{1}(H)=G(L)$. Let $u=\left(u_{1}, u_{2}\right) \in G(R)$.

(a) If $|G(L)| \geq 4$ then $\pi_{1}\left(D_{R}\langle 1,-u\rangle \cap H\right)=D_{L}\left\langle 1,-u_{1}\right\rangle$.

(b) If $L=\mathbb{Z}$ and $\pi_{1}\left(D_{R}\langle 1,-u\rangle \cap H\right) \neq D_{L}\left\langle 1,-u_{1}\right\rangle$ for some $u$, then $H=1 \times H_{2} \cup-1 \times-H_{2}$, where $1 \times H_{2}=H \cap\left(1 \times G\left(R_{2}\right)\right)$ and $H_{2}$ is an ordering on $R_{2}$.

Proof. Suppose that $\pi_{1}\left(D_{R}\langle 1,-u\rangle \cap H\right)=K<D_{L}\left\langle 1,-u_{1}\right\rangle$.

Claim. If $v \in G(L) \backslash K$ then $(v,-1) \in H$.

Since $\pi_{1}(H)=G(L)$ there exists a $w \in G\left(R_{2}\right)$ such that $(v, w)=h \in H$. Now:

$$
\begin{aligned}
D_{R}\langle 1, u h,-h\rangle= & -u\left(D_{L}\langle\langle-u,-v\rangle\rangle^{\prime} \times D_{R_{2}}\left\langle\left\langle-u_{2},-w\right\rangle\right\rangle^{\prime}\right) \\
& \supset u_{1} G(L)^{\cdot} \times u_{2} T,
\end{aligned}
$$

where $T=-D_{R_{2}}\left\langle\left\langle-u_{2},-w\right\rangle\right\rangle^{\prime}$. Also:

$$
\bigcup_{t \in h\left(D_{R}\langle 1,-u\rangle \cap H\right)} D_{R}\langle 1,-t\rangle \subset\left(\bigcup_{k \in K} D_{L}\langle 1,-v k\rangle\right) \times G\left(R_{2}\right) .
$$

Now $v \notin K$ implies $G(L) \neq \cup D_{L}\langle 1,-v k\rangle$ by (3.1). Choose a $g \in G(L) \backslash$ $\cup D_{L}\langle 1,-v k\rangle$.

We check that we may assume $g \neq u_{1}$. If $v \in D_{L}\left\langle 1,-u_{1}\right\rangle$ then we have $u_{1} D_{L}\langle 1,-v\rangle=D_{L}\langle 1,-v\rangle \subset \cup D_{L}\langle 1,-v k\rangle$ and so no $g \in G(L) \backslash \cup D_{L}\langle 1,-v k\rangle$ is equal to $u_{1}$. If instead $v \notin D_{L}\left\langle 1,-u_{1}\right\rangle$ then, as $|K|<\left|D_{L}\left\langle 1,-u_{1}\right\rangle\right|$, there exists a $w \neq u_{1}$ such that $K \subset D_{L}\left\langle 1,-u_{1}\right\rangle \cap D_{L}\langle 1,-w\rangle$. If $v \notin D_{L}\langle 1,-w\rangle$ then $w$ is not in any $D_{L}\langle 1,-v k\rangle$, for $k \in K$, and we may take $g=w$. If $v \in D_{L}\langle 1,-w\rangle$ then $v \notin D_{L}\left\langle 1,-u_{1} w\right\rangle$ and we may take $g=u_{1} w$.

We thus have $g \in u_{1} G(L)^{\cdot} \backslash \cup D_{L}\langle 1,-v k\rangle$. So $g \times u_{2} T \subset u H$ by (3.3). Hence $u_{1} g \times T \subset H$. Then $\left(u_{1} g, u_{2}\right),\left(u_{1} g,-u_{2} w\right) \in H$ and so $(1,-w) \in H$. We obtain that $(v,-1)=(v, w)(1,-w) \in H$ and the Claim is proven.

Now suppose $u_{1} \neq 1$. Let $x \in G(L)^{\text {. }}$. Since $|K|<\left|D_{L}\left\langle 1,-u_{1}\right\rangle\right|$ we have $|K| \leq \frac{1}{4}|G(L)|$. So we can choose $v \in G(L) \backslash\{1, x\} K$. Then $(v,-1)$ and $(v x,-1)$ are in $H$ by the Claim. Hence $(x, 1) \in H$. This shows that $G(L) \times$ $1 \subset H$. But then $D_{L}\left\langle 1,-u_{1}\right\rangle \times 1 \subset D_{R}\langle 1,-u\rangle \cap H$ and $\pi_{1}\left(D_{R}\langle 1,-u\rangle \cap H\right)=$ $D_{L}\left\langle 1,-u_{1}\right\rangle$, as desired.

Next suppose $u_{1}=1$ and $|G(L)| \geq 4$. We show $\pi_{1}\left(D_{R}\langle 1,-u\rangle \cap H\right)=G(L)$.

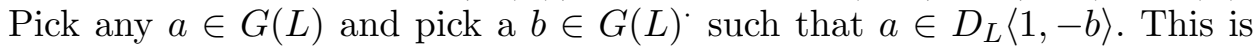
possible since $|G(L)| \geq 4$ implies there are at least two $b$ 's such that $a \in$ 
$D_{L}\langle 1,-b\rangle$. So there is such a $b$ not equal to 1 . Then, by the above paragraph, $\pi_{1}\left(D_{R}\left\langle 1,-\left(b, u_{2}\right)\right\rangle \cap H\right)=D_{L}\langle 1,-b\rangle$ contains $a$. Thus there exists a $k \in$ $D_{R_{2}}\left\langle 1,-u_{2}\right\rangle$ such that $(a, k) \in H$. Thus $(a, k) \in D_{R}\left\langle 1,-\left(u_{1}, u_{2}\right)\right\rangle$, as $u_{1}=1$, and so $a \in \pi_{1}\left(D_{R}\langle 1,-u\rangle \cap H\right)$.

Lastly, suppose $u_{1}=1$ and $L=\mathbb{Z}$. Here $K=\{1\}$ and $v=-1$. The Claim shows that $(-1,-1) \in H$. Now $H_{2}=H \cap\left(1 \times G\left(R_{2}\right)\right)$ has index 2 in $H$ since $1 \times G\left(R_{2}\right)$ has index 2 in $G(R)$ and $H \not \subset 1 \times G\left(R_{2}\right)$. Hence $H=1 \times H_{2} \cup-1 \times-H_{2}$. The last paragraph of the proof of the Claim gives $u_{1} g \times T \subset H$, where $g \neq u_{1}$. Thus $g=-1$ and after multiplying by $-1 \in H$ we get:

$$
1 \times D_{R_{2}}\left\langle\left\langle-u_{2},-w\right\rangle\right\rangle^{\prime} \subset H .
$$

This holds for all $w \in G\left(R_{2}\right)$ such that $(-1, w) \in H$, that is, for all $w \in-H_{2}$. Thus for any $h_{2} \in H_{2}$ :

$$
\begin{aligned}
-u_{2} D_{R_{2}}\left\langle 1, h_{2}\right\rangle & \subset D_{R_{2}}\left\langle-u_{2}, h_{2},-u_{2} h_{2}\right\rangle \subset H_{2} \\
D_{R_{2}}\left\langle 1, h_{2}\right\rangle & \subset H_{2} .
\end{aligned}
$$

Thus $H_{2}$ is a preordering. Also (5.3) holds for any $u_{2} \in G\left(R_{2}\right)$ with $\pi_{1}\left(D_{R}\left\langle 1,-\left(1, u_{2}\right)\right\rangle\right)=1$. That is, $D_{R}\left\langle 1,-\left(1, u_{2}\right)\right\rangle \cap\left(-1 \times-H_{2}\right)=\emptyset$ or equivalently, $u_{2}$ is not in $D_{R_{2}}\left\langle 1, h_{2}\right\rangle$ for any $h_{2} \in H_{2}$. For such a $u_{2},(5.3)$ implies $-u_{2} \in H_{2}$. Hence:

$$
G\left(R_{2}\right)=-H_{2} \cup \bigcup_{h_{2} \in H_{2}} D_{R_{2}}\left\langle 1, h_{2}\right\rangle .
$$

But $H_{2}$ is a preordering so that $\cup D_{R_{2}}\left\langle 1, h_{2}\right\rangle \subset H_{2}$. Thus $G\left(R_{2}\right)=-H_{2} \cup H_{2}$, $\mathrm{H}_{2}$ has index 2 in $G\left(R_{2}\right)$ and so $H_{2}$ is an ordering.

Notation. Suppose $R=R_{1} \sqcap R_{2}$ and that $H$ is a subgroup of $G(R)$. For $x \in G\left(R_{1}\right)$ set $F(x)=\left\{y \in G\left(R_{2}\right):(x, y) \in H\right\}$.

Lemma 5.4. Let $R=R_{1} \sqcap R_{2}$ and let $S$ be an $H$-extension of $R$. Let $\pi_{1}$ be the projection of $G(R)$ onto $G\left(R_{1}\right)$ and suppose $\pi_{1}(H)=G\left(R_{1}\right)$. Then for all $x \in G\left(R_{1}\right)$ :

(a) $F(x)$ is non-empty.

(b) $F(1)$ is a subgroup of $G\left(R_{1}\right)$.

(c) $F(x)$ is a coset of $F(1)$.

Proof. No $F(x)$ is empty since $\pi_{1}(H)=G\left(R_{1}\right)$. Clearly $F(1)$ is a subgroup. Fix $y_{0} \in F(x)$. If $y \in F(1)$ then $(1, y),\left(x, y_{0}\right) \in H$ implies $\left(x, y y_{0}\right) \in H$ and so $y y_{0} \in F(x)$. This says $y_{0} F(1) \subset F(x)$.

Now let $y \in F(x)$. Then $\left(x, y_{0}\right),(x, y) \in H$ so that $\left(1, y y_{0}\right) \in H$. Hence $y y_{0} \in F(1)$ and we have the reverse inclusion $F(x) \subset y_{0} F(1)$. 
Lemma 5.5. Let $R=R_{1} \sqcap R_{2}$ be of elementary type. Let $S$ be an $H$ extension of $R$. Let $\pi_{1}$ be the projection of $G(R)$ onto $G\left(R_{1}\right)$. Suppose the following:

(1) $\pi_{1}(H)=G\left(R_{1}\right)$.

(2) $F(a) \cap \operatorname{rad}\left(R_{2}\right) \subset\{1\}$, for all $a \in G\left(R_{1}\right)$.

(3) For all $u=\left(u_{1}, u_{2}\right) \in G(R)$ we have $\pi_{1}\left(D_{R}\langle 1,-u\rangle \cap H\right)=D_{R_{1}}\left\langle 1,-u_{1}\right\rangle$. Then $H=G\left(R_{1}\right) \times H_{2}$, for some subgroup $H_{2} \subset G\left(R_{2}\right)$.

Proof. Let $a \in G\left(R_{1}\right)$. We will first show that:

$$
G\left(R_{2}\right)=\bigcup_{k \in F(a)} D_{R_{2}}\langle 1,-k\rangle \text {. }
$$

Pick any $b \in D_{R_{1}}\langle 1,-a\rangle$ and any $g \in G\left(R_{2}\right)$. Then $a \in D_{R_{1}}\langle 1,-b\rangle=$ $\pi_{1}\left(D_{R}\langle 1,-(b, g)\rangle \cap H\right)$ by assumption (3). Hence there exists a $k \in D_{R_{2}}\langle 1,-g\rangle$ with $(a, k) \in H$. That is, $g \in D_{R_{2}}\langle 1,-k\rangle$ for some $k \in F(a)$, proving (5.6).

Write $F(a)=y F(1)$ as in (5.4). Then (5.6) becomes:

$$
G\left(R_{2}\right)=\bigcup_{k \in F(1)} D_{R_{2}}\langle 1,-y k\rangle .
$$

Thus $y \in \operatorname{rad}\left(R_{2}\right) \cdot F(1)$ by (3.1). That is, there exists a $d \in \operatorname{rad}\left(R_{2}\right)$ such that $d \in y F(1)=F(a)$. By assumption (2) then $d=1$. Hence $y \in F(1)$ and so $F(a)=F(1)$. By assumption (1) we have $(a, m) \in H$ for some $m \in G\left(R_{2}\right)$. Then $m \in F(a)=F(1)$ so that $(1, m) \in H$ also. So $(a, 1)=(a, m)(1, m) \in$ $H$. Hence $G\left(R_{1}\right) \times 1 \subset H$ and $H=G\left(R_{1}\right) \times F(1)$.

We first complete the case of a local factor $L$ with $|G(L)| \geq 8$.

Corollary 5.7. Let $R=L \sqcap R_{2}$, with $R_{2}$ of elementary type, $L$ of local type and $|G(L)| \geq 8$. Let $S$ be an $H$-extension of $R$. Suppose $F(a) \cap \operatorname{rad}\left(R_{2}\right) \subset\{1\}$ for all $a \in G(L)$. Then either $H=1 \times H_{2}$ or $H=G(L) \times H_{2}$ for some subgroup $\mathrm{H}_{2} \subset G\left(R_{2}\right)$.

Proof. Again let $\pi_{1}$ denote the projection of $G(R)$ onto $G(L)$. We know that $\pi_{1}(H)=1$ or $G(L)$, by (2.2). If $\pi_{1}(H)=1$ then clearly $H=1 \times H_{2}$ for some subgroup $H_{2}$. So suppose that $\pi_{1}(H)=G(L)$, the first hypothesis of (5.5). We are assuming the second hypothesis as well. And (5.2) shows the third hypothesis of (5.5) holds. Hence $H=G(L) \times H_{2}$, for some subgroup $\mathrm{H}_{2}$.

The argument for $R=\mathbb{Z} \sqcap R_{2}$ is different.

Lemma 5.8. Let $R$ be a real Witt ring of elementary type. Let $P \subset G(R)$ be an ordering. Suppose that for all $x \in P$ that:

$$
P=\bigcup_{k \in D\langle 1,-x\rangle \cap P} D\langle 1, x k\rangle .
$$

Then $R=\mathbb{Z} \sqcap R_{2}$, for some Witt ring $R_{2}$. 
Proof. Suppose $\mathbb{Z}$ is not a factor of $R$. Then $R$ has a group ring factor that is real. Thus $R=R_{0}\left[E_{1}\right] \sqcap R_{2}$, for some Witt rings $R_{0}, R_{2}$, and we may assume $P=P_{0}\{1, t\} \times G\left(R_{2}\right)$, where $P_{0} \subset G\left(R_{0}\right)$ is an ordering on $R_{0}$ and $E_{1}=\{1, t\}$. Then take $x=(t, 1)$. We have that $D\langle 1,-x\rangle=\{1,-t\} \times G\left(R_{2}\right)$ and $D\langle 1,-x\rangle \cap P=1 \times G\left(R_{2}\right)$. Thus:

$$
\begin{aligned}
P=\bigcup_{k \in D\langle 1,-x\rangle \cap P} D\langle 1, x k\rangle & =\bigcup_{g_{2} \in G\left(R_{2}\right)} D\left\langle(1,1),\left(t, g_{2}\right)\right\rangle \\
& =\{1, t\} \times G\left(R_{2}\right) .
\end{aligned}
$$

Hence $P_{0}=1$ and $R_{0}=\mathbb{Z}$, giving a contradiction.

Lemma 5.9. Let $R=\mathbb{Z} \sqcap R_{2}$ and suppose $S$ is an $H$-extension of $R$. Then one of the following occurs.

(a) $H=1 \times H_{2}$ for some subgroup $H_{2} \subset G\left(R_{2}\right)$.

(b) $R=\mathbb{Z} \sqcap R_{3}$, for some Witt ring $R_{3}$, and $\{ \pm 1\} \times 1 \subset H$.

(c) $R=\mathbb{Z} \sqcap \mathbb{Z} \sqcap R_{3}$, for some Witt ring $R_{3}$, and $(1,1) \times G\left(R_{3}\right) \subset H$.

Proof. Again let $\pi_{1}$ be the projection of $G(R)$ onto $G(\mathbb{Z})=\{ \pm 1\}$. If $\pi_{1}(H)=$ 1 the we are in case (a). Thus we may assume that $\pi_{1}(H)=G(\mathbb{Z})$. If for every $u \in G(R)$ we have that $\pi_{1}\left(D_{R}\langle 1,-u\rangle \cap H\right)=D\left\langle 1,-\pi_{1}(u)\right\rangle$ then (5.5) implies we are in case (b). So suppose this fails for some $u \in G(R)$. Then by (5.2) $H=1 \times H_{2} \cup\left(-1 \times-H_{2}\right)$, for some ordering $H_{2}$ of $G\left(R_{2}\right)$. We will first show that for every $h_{2} \in H_{2}$ that:

$$
H_{2}=\bigcup_{k \in D_{R_{2}}\left\langle 1,-h_{2}\right\rangle \cap H_{2}} D_{R_{2}}\left\langle 1, h_{2} k\right\rangle .
$$

Consider $\varphi=\left\langle(1,1),\left(1, h_{2}\right),(1,-1)\right\rangle \in S$. We compute its value set two ways. First:

$$
D_{S}\left\langle(1,1),\left(1, h_{2}\right),(1,-1)\right\rangle=\bigcup_{\beta \in D_{S}\left\langle(1,1),\left(1,-h_{2}\right)\right\rangle} D_{S}\left\langle(1,1), \beta\left(1, h_{2}\right)\right\rangle .
$$

Now $\left(-1, h_{2}\right) \notin H$ so $D_{S}\left\langle(1,1),\left(1,-h_{2}\right)\right\rangle=1 \times D_{R_{2}}\left\langle 1,-h_{2}\right\rangle$. For $\varphi$ to represent an element of $\alpha\left(1 \times G\left(R_{2}\right)\right)$ we must have $\beta \in-H=H$. That is, $\beta=\left(1, \beta_{2}\right)$ with $\beta_{2} \in H_{2}$. Thus:

$$
D_{S}(\varphi) \cap \alpha\left(1 \times G\left(R_{2}\right)\right)=\alpha \cdot \underset{\beta_{2} \in D_{R_{2}}\left\langle 1,-h_{2}\right\rangle \cap H_{2}}{\bigcup_{R_{2}}}\left(1 \times D_{R_{2}}\left\langle 1, \beta_{2} h_{2}\right\rangle\right) .
$$

Next:

$$
D_{S}(\varphi)=\left(1, h_{2}\right) \cdot \bigcup_{\gamma \in D_{S}\langle(1,1),(1,-1)\rangle} D_{S}\left\langle(1,1), \gamma\left(1, h_{2}\right)\right\rangle .
$$


For any $x \in H_{2}$ take $\gamma=(1, x) \in D_{S}\langle(1,1),(1,-1)\rangle$. Then since $\left(-1,-x h_{2}\right) \in$ $H$ :

$$
\begin{aligned}
\alpha(1, x) & \in\left(1, h_{2}\right) D_{S}\left\langle(1,1),-\left(-1,-x h_{2}\right)\right\rangle \\
& =\left(1, h_{2}\right) \cdot\{1, \alpha\}\left(1 \times D_{R_{2}}\left\langle 1, x h_{2}\right\rangle\right) .
\end{aligned}
$$

Thus $D_{S}(\varphi) \cap \alpha\left(1 \times G\left(R_{2}\right)\right)=\alpha\left(1 \times H_{2}\right)$. The two computations of $D_{S}(\varphi)$ thus yield $H_{2}=\cup D_{R_{2}}\left\langle 1, \beta h_{2}\right\rangle$, over $\beta \in D_{R_{2}}\left\langle 1,-h_{2}\right\rangle \cap H_{2}$.

We may now apply (5.8) to obtain $R_{2}=\mathbb{Z} \sqcap R_{3}$, for some Witt ring $R_{3}$. Let $H_{3} \subset G\left(R_{3}\right)$ be the subgroup such that $H_{2} \cap\left(1 \times G\left(R_{3}\right)\right)=1 \times H_{3}$. We note that both $H_{2}$ and $1 \times G\left(R_{3}\right)$ have index two in $G\left(R_{2}\right)$.

If $H_{2}=1 \times G\left(R_{3}\right)$ then $(1,1) \times G\left(R_{3}\right) \subset H$ and we are in case (c). So suppose $H_{2} \neq 1 \times G\left(R_{3}\right)$. Then $1 \times H_{3}$ has index two in $H_{2}$ and $H_{3}$ has index two in $G\left(R_{3}\right)$. Write $H_{2}=1 \times H_{3} \cup\left(-1 \times z H_{3}\right)$, for some $z \in G\left(R_{3}\right)$. Then:

(5.10)

$H=\left[(1,1) \times H_{3}\right] \cup\left[(1,-1) \times z H_{3}\right] \cup\left[(-1,-1) \times-H_{3}\right] \cup\left[(-1,1) \times-z H_{3}\right]$.

Now $\left[G\left(R_{3}\right): H_{3}\right]=2$ implies at least one of the cosets $z H_{3},-H_{3},-z H_{3}$ equals $H_{3}$. Say $z H_{3}=H_{3}$. Then the second term of $(5.10)$ shows $(1,1,1)$, $(1,-1,1) \in H$. Set $R_{4}$ equal to the product of the first copy of $\mathbb{Z}$ and $R_{3}$. Then $R=\mathbb{Z} \sqcap R_{4}$ and $\{ \pm 1\} \times 1 \subset H$. We are thus in case (b). Next say $-H_{3}=H_{3}$. Then $(1,-1) \in 1 \times H_{3} \subset H_{2}$. Since $H_{2}$ is an ordering we have $D_{R_{2}}\langle(1,1),(1,-1)\rangle \subset H_{2}$. But the $1 \times G\left(R_{3}\right) \subset H_{2}$, a case we have already considered. Lastly, suppose $-z H_{3}=H_{3}$. Then the fourth term of (5.10) shows $(1,1,1),(-1,1,1) \in H$. This is case (b) again.

\section{Products: Group ring factors.}

Lemma 6.1. Let $R=R_{1} \sqcap R_{2}$, with $R_{1}=R_{0}\left[E_{1}\right]$ and $E_{1}$ generated by $t$. Let $S$ be an $H$-extension of $R$. Let $\pi_{1}$ be the projection of $G(R)$ onto $G\left(R_{1}\right)$ and suppose $\pi_{1}(H) \not \subset G\left(R_{0}\right)$. Then either $\pi_{1}(H)=G\left(R_{1}\right)$ or $1 \times G\left(R_{2}\right) \subset H$.

Proof. From $\pi_{1}(H) \not \subset G\left(R_{0}\right)$ we may assume $h=\left(t, g_{2}\right) \in H$, for some $g_{2} \in G\left(R_{2}\right)$. Suppose $\pi_{1}(H) \neq G\left(R_{1}\right)$, Choose $-g_{1} \in G\left(R_{1}\right) \backslash \pi_{1}(H)$. Then $-g_{1} t \notin \pi_{1}(H)$. Set $u=\left(g_{1} t, 1\right)$ and note that $\pi_{1}\left(D_{R}\langle 1,-u\rangle \cap H\right)=1$. Now:

$$
\begin{aligned}
D_{R}\langle 1, u h,-h\rangle= & D_{R_{1}}\left\langle 1, g_{1},-t\right\rangle \times D_{R_{2}}\left\langle 1, g_{2},-g_{2}\right\rangle \\
& \supset g_{1} \times G\left(R_{2}\right) .
\end{aligned}
$$

Also:

$$
\bigcup_{k \in h\left(D_{R}\langle 1,-u\rangle \cap H\right)} D_{R}\langle 1,-k\rangle \subset D_{R_{1}}\langle 1,-t\rangle \times G\left(R_{2}\right)=\{1,-t\} \times G\left(R_{2}\right) .
$$

Hence by (3.3), $g_{1} \times G\left(R_{2}\right) \subset u H$. Multiplying by $u$ gives $t \times G\left(R_{2}\right) \subset H$. Thus $1 \times G\left(R_{2}\right) \subset H$. 
Lemma 6.2. Let $R=R_{1} \sqcap R_{2}$, with $R_{1}=R_{0}\left[E_{1}\right]$ and $E_{1}$ generated by t. Let $S$ be an $H$-extension of $R$. Let $\pi_{1}$ be the projection of $G(R)$ onto $G\left(R_{1}\right)$ and suppose $\pi_{1}(H)=G\left(R_{1}\right)$. If $u_{1} \in G\left(R_{0}\right)$ and $u=\left(u_{1}, u_{2}\right)$ then $\pi_{1}\left(D_{R}\langle 1,-u\rangle \cap H\right)=D_{R_{1}}\left\langle 1,-u_{1}\right\rangle$.

Proof. Set $K=\pi_{1}\left(D_{R}\langle 1,-u\rangle \cap H\right)$ and suppose $K<D_{R_{1}}\left\langle 1,-u_{1}\right\rangle$. Let $g \in G\left(R_{0}\right)$. Then $\left(g t, g_{2}\right) \in H$ for some $g_{2} \in G\left(R_{2}\right)$, since $\pi_{1}(H)=G\left(R_{1}\right)$. Now $D_{R}\langle 1, u h,-h\rangle$ contains $-g t D_{R_{1}}\left\langle 1,-u_{1}\right\rangle \times u_{2} T$, where $T=$ $-D_{R_{2}}\left\langle\left\langle-u_{2},-g_{2}\right\rangle\right\rangle^{\prime}$. Also:

$$
\begin{aligned}
\bigcup_{w \in h\left(D_{R}\langle 1,-u\rangle \cap H\right)} D_{R}\langle 1,-w\rangle & \subset \bigcup_{k \in K} D_{R_{1}}\langle 1,-k g t\rangle \times G\left(R_{2}\right) \\
& =(\{1\} \cup-g t K) \times G\left(R_{2}\right) .
\end{aligned}
$$

Hence by (3.3), if $y \in D_{R_{1}}\left\langle 1,-u_{1}\right\rangle \backslash K$ then:

$$
\begin{aligned}
& -g t y \times u_{2} T \subset u H \\
& -g t u_{1} y \times T \subset H .
\end{aligned}
$$

Now $u_{2}$ and $-u_{2} g_{2}$ are in $T$ so $\left(-g t u_{1} y, u_{2}\right)$ and $\left(-g t u_{1} y,-u_{2} g_{2}\right)$ are in $H$. Thus $\left(1,-g_{2}\right) \in H$ and as result $(g t,-1) \in H$.

This holds for all $g \in G\left(R_{0}\right)$ so we have that $t G\left(R_{0}\right) \times-1 \subset H$. Thus $G\left(R_{0}\right) \times 1 \subset H$. But the $D_{R_{1}}\left\langle 1,-u_{1}\right\rangle \times 1 \subset D_{R}\langle 1,-u\rangle \cap H$ and $\pi_{1}\left(D_{R}\langle 1,-u\rangle \cap\right.$ $H)=D_{R_{1}}\left\langle 1,-u_{1}\right\rangle$, a contradiction.

Lemma 6.3. Let $R=R_{1} \sqcap R_{2}$, with $R_{1}=R_{0}\left[E_{1}\right]$ non-degenerate and $E_{1}$ generated by $t$. Let $S$ be an $H$-extension of $R$. Let $\pi_{1}$ be the projection of $G(R)$ onto $G\left(R_{1}\right)$ and suppose $\pi_{1}(H)=G\left(R_{1}\right)$. Then $G\left(R_{1}\right) \times 1 \subset H$.

Proof. We will first show $F(g)=F(1)$ for all $g \in G\left(R_{0}\right)$. Let $g \in G\left(R_{0}\right)$.

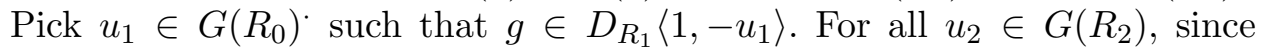
$g \in D_{R_{1}}\left\langle 1,-u_{1}\right\rangle=\pi_{1}\left(D_{R}\left\langle 1,-\left(u_{1}, u_{2}\right)\right\rangle \cap H\right)$, there exists a $k \in G\left(R_{2}\right)$ with $(g, k) \in H$ and $k \in D_{R_{2}}\left\langle 1,-u_{2}\right\rangle$. That is, $G\left(R_{2}\right)=\cup_{k \in F(g)} D_{R_{2}}\langle 1,-k\rangle$. By (3.1) and (5.4), $F(g)=F(1)$.

We next show $G\left(R_{0}\right) \times 1 \subset H$. Continue to let $g \in G\left(R_{0}\right)$. Now, as $g \in \pi_{1}(H)=G\left(R_{1}\right)$, we have $(g, m) \in H$ for some $m \in G\left(R_{2}\right)$. Then $m \in F(g)=F(1)$ so $(1, m) \in H$ and hence $(g, 1)=(g, m)(1, m) \in H$. This shows $G\left(R_{0}\right) \subset H$.

We will be done if we show $F(t)=F(1)$. Then, if $(t, k) \in H$ we get $(1, k)$ and hence $(t, 1)$ are in $H$. Apply the previous paragraph to get $G\left(R_{1}\right) \times 1=$ $\{1, t\} G\left(R_{0}\right) \times 1 \subset H$.

So suppose $F(t) \neq F(1)$. We have by (3.1):

$$
G\left(R_{2}\right) \neq \bigcup_{k \in F(t)} D_{R_{2}}\langle 1,-k\rangle \text {. }
$$

Pick $u_{2} \in G\left(R_{2}\right) \backslash \cup D_{R_{2}}\langle 1,-k\rangle$. Set $u=\left(-t, u_{2}\right)$. Then, as there is no $k$ with $(t, k) \in H$ and $k \in D_{R_{2}}\langle 1,-k\rangle$, we have $\pi_{1}\left(D_{R}\langle 1,-u\rangle \cap H\right)=1$. Pick 
any $g \in G\left(R_{0}\right)^{\text {' (we note that }}\left|G\left(R_{0}\right)\right|>1$, else $R_{1}$ is degenerate). Pick any $g_{2} \in F(g)$ and set $h=\left(g, g_{2}\right) \in H$. Now:

$$
\begin{aligned}
D_{R}\langle 1, u h,-h\rangle= & D_{R_{1}}\langle 1,-g t,-g\rangle \times D_{R_{2}}\left\langle 1, u_{2} g_{2},-g_{2}\right\rangle \\
& \supset-g t \times D_{R_{2}}\left\langle 1, u_{2} g_{2},-g_{2}\right\rangle .
\end{aligned}
$$

Also:

$$
\bigcup_{w \in h\left(D_{R}\langle 1,-u\rangle \cap H\right)} D_{R}\langle 1,-w\rangle \subset D_{R_{1}}\langle 1,-g\rangle \times G\left(R_{2}\right) .
$$

Hence, by (3.3),

$$
\begin{aligned}
-g t \times D_{R_{2}}\left\langle 1, u_{2} g_{2},-g_{2}\right\rangle & \subset u H \\
-t \times D_{R_{2}}\left\langle g_{2}, u_{2},-1\right\rangle & \subset H .
\end{aligned}
$$

In particular, $\left(-t, g_{2}\right) \in H$. Since $(-1,1) \in G\left(R_{0}\right) \times 1 \subset H$, we get $\left(t, g_{2}\right) \in$ $H$. But then $g_{2} \in F(t) \cap F(g)$, which equals $F(t) \cap F(1)$ by previous work. $F(t)$ is a coset of $F(1)$, by (5.4), so in fact $F(t)=F(1)$ as desired.

\section{The Main Theorem.}

Theorem 7.1. Let $R$ be a Witt ring of elementary type. If $S$ is an $H$ extension of $R$, for some subgroup $H \subset G(R)$, then $S$ is also of elementary type.

Proof. We argue by induction on $|G(R)|$. If $|G(R)| \leq 2$ then either $H=1$ or $H=G(R)$ and we are done by (1.1). Suppose $|G(R)|>2$. If $R=A \sqcap B$ then $\pi_{A}$ will denote the projection of $G(R)$ onto $G(A)$.

Now suppose $R$ is degenerate. Write $R=D_{k} \sqcap R_{2}$, where $R_{2}$ is nondegenerate and $G\left(D_{k}\right) \times 1=\operatorname{rad}(R)$. If some $d \in \operatorname{rad}(R) \cap H$ then write $R=D_{1} \sqcap R_{3}$, where $d$ generates $D_{1}$. We have $G\left(D_{1}\right) \times 1 \subset H$ and so $H=G\left(D_{1}\right) \times H_{3}$, for some subgroup $H_{3} \subset G\left(R_{3}\right)$. Then (3.2) implies $S=D_{1} \sqcap S_{3}$, where $S_{3}$ is an $H_{3}$-extension of $R_{3}$. By induction, $S_{3}$ is of elementary type and so $S$ is also. We may thus assume $\operatorname{rad}(R) \cap H=1$. If $\pi_{D_{k}}(H) \cap G\left(D_{k}\right) \neq 1$ then by (4.1) we may replace $H$, without affecting $S$, by another subgroup $H_{0}$ such that $\pi_{D_{k}}\left(H_{0}\right) \cap G\left(D_{k}\right)=1$. We assume this has already been done so that $\pi_{D_{k}}(H) \cap G\left(D_{k}\right)=1$. We note this also holds trivially if $R$ is non-degenerate and $k=0$.

Next suppose $R$ has a local type factor $L$ with $|G(L)| \geq 8$. Write $R=L \sqcap$ $R_{4}$. $D_{k}$ is a factor of $R_{4}$. We check the hypothesis of (5.7). Let $a \in G(L)$ and suppose $x \in F(a) \cap \operatorname{rad}\left(R_{4}\right)$. This means $(a, x) \in H$ and so $x \in \pi_{D_{k}}(H)=1$. Thus $F(a) \cap \operatorname{rad}\left(R_{4}\right) \subset\{1\}$, as desired. Apply (5.6) to get that either $G(L) \times 1 \subset H$ or $\pi_{L}(H)=1$. In the first case, $H=G(L) \times H_{4}$, for some subgroup $H_{4}$ of $G\left(R_{4}\right)$. Then (3.2) implies $S=L \sqcap S_{4}$, where $S_{4}$ is an $H_{4^{-}}$ extension of $R_{4}$. By induction, $S_{4}$ is of elementary type and so $S$ is also. We may thus assume we are in the second case: $\pi_{L}(H)=1$. 
Now suppose the local factor is $L_{1}=\mathbb{Z}$. There are three cases according to (5.9). In case (b) we can write $R=\mathbb{Z} \sqcap R_{5}$, with $\{ \pm 1\} \times 1 \subset H$. Then $H=G(\mathbb{Z}) \times H_{5}$, for some subgroup $H_{5} \subset G\left(R_{5}\right)$. Applying (3.2) again gives $S=\mathbb{Z} \sqcap S_{5}$, where $S_{5}$ is an $H_{5}$-extension of $R_{5}$. Induction again shows $S$ is of elementary type. In case (c) we can write $R=\mathbb{Z} \sqcap \mathbb{Z} \sqcap R_{6}$, with $(1,1) \times G\left(R_{6}\right) \subset H$. Once again (3.2) yields $S=S_{0} \sqcap R_{6}$, where $S_{0}$ is an $H_{0^{-}}$ extension of $\mathbb{Z} \sqcap \mathbb{Z}$, for some subgroup $H_{0}$. Since $\mathbb{Z} \sqcap \mathbb{Z} \cong \mathbb{Z}\left[E_{1}\right]$, (1.5) shows $S_{0}$ is of elementary type (in (1.5)(b) we have $R_{0}=\mathbb{Z}$ so that its extension is of elementary type as $\left.\left|G\left(R_{0}\right)\right|=2\right)$. Thus $S$ is also of elementary type. We may thus assume we are in case (a) of (5.9), namely, that $\pi_{L_{1}}(H)=1$. This is the same conclusion as when the local factor has at least 8 square classes.

The only local type factors we have omitted are those with 4 square classes and these Witt rings are group rings. We are thus in the following position: $R=Y \sqcap W_{1} \sqcap \ldots \sqcap W_{n}$, where $Y$ is a product of $D_{k}$ with $k \geq 0$, and local type rings $L$ with $|G(L)| \neq 4$ and each $W_{i}$ is a non-degenerate group ring. (It is possible that $Y=1$.) We also have $\pi_{Y}(H)=1$, so that if $n=0$ then $H=1$ and we are done by (1.1). So suppose $n \geq 1$. Write $W_{i}=V_{i}\left[E_{1}\right]$. We first suppose that $\pi_{W_{i}}(H) \not \subset G\left(V_{i}\right)$ for some $i$. Write $R=W_{i} \sqcap R_{7}$. There are two possibilities according to $(6.1)$.

The first possibility is that $1 \times G\left(R_{7}\right) \subset H$. Write $H=H_{0} \times G\left(R_{7}\right)$, for some subgroup $H_{0}$ of $G\left(W_{i}\right)$. Then (3.2) gives that $S=S_{0} \sqcap R_{7}$, for $S_{0}$, some $H_{0}$-extension of $W_{i}$. If $G\left(R_{7}\right) \neq 1$ then we are done by induction. We drop the subscript $i$ and suppose then that $R=W=W_{0}\left[E_{n}\right]$, where $W_{0}$ is basic and $n \geq 1$. In cases (a),(c),(d) we have $S$ is of elementary type. In case (b) $S=S_{0}\left[E_{n}\right]$, where $S_{0}$ is an $H$-extension of $W_{0}$, and so again $S$ is of elementary type by induction.

The second possibility in (6.1) is that $\pi_{W_{i}}(H)=G\left(W_{i}\right)$. Then by (6.3) we have $H=G\left(W_{i}\right) \times H_{7}$, where $H_{7}$ is a subgroup of $R_{7}$. Apply (3.2) once again to get that $S=W_{i} \sqcap S_{7}$, where $S_{7}$ is an $H_{7}$-extension of $R_{7}$. Induction gives that $S$ is of elementary type. This completes the result when (6.1) applies, that is, when $\pi_{W_{i}}(H) \not \subset G\left(V_{i}\right)$, for some $i$.

We may thus assume we have $R=Y \sqcap W_{1} \sqcap \ldots \sqcap W_{n}$, with $\pi_{Y}(H)=1$ and every $\pi_{W_{i}}(H) \subset V_{i}$. Choose $t_{i} \notin V_{i}$, for each $i$ and set $g=\left(1, t_{1}, \ldots, t_{n}\right) \in$ $G(R)$. Then:

$$
g \notin \pm \bigcup_{h \in H^{*}} D_{R}\langle 1,-h\rangle
$$

as for any $h \in H^{\cdot}$ has a coordinate in $G\left(V_{i}\right)^{\prime}$. By (1.2), $g \notin B(R)$. Thus $R$ is itself a group ring, a case we covered two paragraphs ago.

The previous sections can be used to determine the possible $H$-extensions of a given ring $R$. As an example, consider $R=\left(D_{1} \sqcap L_{3}\right)\left[E_{2}\right]$. Let $d$ generate $D_{1},-1, a, b$ generate $L_{3}$ and $s, t$ generate $E_{2}$. Thus $G(R)=\operatorname{gp}(d,-1, a, b, s, t)$, where $\operatorname{gp}(A)$ denotes the group generated by $A$. $G(R)$ has 2825 subgroups, 
47 of which will yield $H$-extensions. Up to isomorphism, there are exactly $8 H$-extensions of $R$. Below we list the 8 extensions $S$ along with one choice for the corresponding subgroup $H$.

$\begin{array}{ll}\text { 1. }\left(D_{1} \sqcap L_{3}\right)\left[E_{3}\right] & 1 \\ \text { 2. }\left(D_{1} \sqcap\left(D_{1} \sqcap L_{3}\right)\left[E_{1}\right]\right)\left[E_{1}\right] & \operatorname{gp}(d,-1, a, b, s) \\ \text { 3. } D_{1} \sqcap\left(D_{1} \sqcap L_{3}\right)\left[E_{2}\right] & G(R) \\ \text { 4. }\left(\mathbb{Z} \sqcap\left(D_{1} \sqcap L_{3}\right)\left[E_{1}\right]\right)\left[E_{1}\right] & \operatorname{gp}(d, a, b, s) \\ \text { 5. } \mathbb{Z} \sqcap\left(D_{1} \sqcap L_{3}\right)\left[E_{2}\right] & \operatorname{gp}(d, a, b, s, t) \\ \text { 6. }\left(D_{1} \sqcap L_{3}\left[E_{1}\right]\right)\left[E_{2}\right] & \operatorname{gp}(d) \\ \text { 7. }\left(D_{2} \sqcap L_{3}\right)\left[E_{2}\right] & \operatorname{gp}(d,-1, a, b) \\ \text { 8. }\left(D_{1}\left[E_{1}\right] \sqcap L_{3}\right)\left[E_{2}\right] & \operatorname{gp}(-1, a, b)\end{array}$

We give a brief sketch of how this list was derived. Begin by running through the cases of (1.5). In (a) $H=1$ and $S$ is (1) by (1.1). In (c), $G\left(R_{0}\right)$ is a proper subgroup of $H$, so $S$ is (2) or (3), depending on whether or not $H=G(R)$. In (d) $G\left(R_{0}\right) \subset \pm H,-1 \notin H$ and $H \not \subset G\left(R_{0}\right)$. Thus $H$ looks like a subgroup $K$ of index 2 in $G\left(R_{0}\right)$ that does not contain -1, together with one or more elements from $\{t, s, t s\} . S$ is (4) if $\left|H \cap E_{2}\right|=2$ and (5) if $\left|H \cap E_{2}\right|=4$. In (1.5)(b) $S=S_{0}\left[E_{2}\right]$, where $S_{0}$ is an $H$-extension of $R_{0}$. Now $R_{0}=D_{1} \sqcap L_{3}$. By (4.1) we can assume that either $H=G\left(D_{1}\right) \times H_{2}$, or that $H=1 \times H_{2}$, for some $H_{2} \subset G\left(L_{3}\right)$. Now $H_{2}=1$ or $H_{2}=G\left(L_{3}\right)$ by (2.3) and (5.7). Since we have already done the case $H=1$ this gives three choices: $G\left(D_{1}\right) \times 1, G\left(D_{1}\right) \times G\left(L_{3}\right)$ and $1 \times G\left(L_{3}\right)$. The corresponding $S$ is (6), (7) and (8), respectively.

\section{References}

[1] A. Carson and M. Marshall, Decomposition of Witt rings, Canad. J. Math., 34 (1982), 1276-1302.

[2] R. Fitzgerald, Witt rings under odd degree extensions, Pac. J. Math., 158 (1993), 121-143.

[3] B. Jacob and R. Ware, Realizing dyadic factors of elementary type Witt rings and pro-2 Galois groups, Math. Z., 208 (1991), 193-208.

[4] M. Marshall, Abstract Witt Rings, Queen's Papers in Pure and Applied Math., 57, Queen's University, 1980.

Received September 29, 1997 and revised March 25, 1998.

SOUTHERN ILlinOIS UNIVERSITY

Carbondale, IL 62901-4408

E-mail address: rfitzg@math.siu.edu 